\title{
Sustainable Transmission Planning in Imperfectly Competitive Electricity Industries: Balancing Economic and Environmental Outcomes
}

\author{
Afzal S. Siddiqui* \\ Department of Statistical Science, University College London, Department of Computer and Systems Sciences, \\ Stockholm University, and Department of Decision Sciences, HEC Montréal \\ Makoto Tanaka \\ National Graduate Institute for Policy Studies (GRIPS) and Research Institute of Economy, Trade and Industry \\ (RIETI) \\ Yihsu Chen \\ Department of Technology Management, University of California Santa Cruz
}

\begin{abstract}
We explore the role of a transmission system operator (TSO) that builds a transmission line to accommodate renewable energy in order to lower emissions as required by government policy. In contrast to central planning, a TSO in a deregulated electricity industry can only indirectly influence outcomes through its choice of the transmission line capacity. Via a bi-level model, we show that this results in less transmission capacity and with limited emissions control in a perfectly competitive industry vis-à-vis a benchmark centrally planned system. A carbon charge on industry that fully accounts for the cost of pollution damage leads to a perfect alignment of incentives and maximised social welfare only under perfect competition. By contrast, a carbon charge may actually lower social welfare under a Cournot oligopoly as the resulting reduction in consumption facilitates the further exercise of market power.
\end{abstract}

Keywords: OR in environment and climate change, transmission planning, bi-level modelling, market power

\section{Introduction}

The challenge of integrating renewable energy (RE) resources in electric power systems in order to mitigate climate change is hampered by the deregulated nature of the electric power industry. Over the past thirty years, power companies have evolved from being state-regulated and vertically integrated entities to facing lighter regulation while competing for market share (Wilson, 2002). In spite of this evolution, transmission-expansion decisions, which largely exhibit natural monopoly

\footnotetext{
${ }^{*}$ Corresponding author

Email address: afzal.siddiqui@ucl.ac.uk (Afzal S. Siddiqui*)
} 
characteristics, have remained under the auspices of regulated planners, viz., transmission system operators (TSOs). ${ }^{1}$

In order to assess the impact of transmission expansion within a market environment, several models have been posited in the literature. Since at least Garver (1970), the use of optimisation models for transmission planning has been known. Taking examples from real-world applications of optimisation to transmission planning, Hobbs (1995) points out their use in case studies involving Seattle City Light and EdF even if transmission interconnections in the real world are difficult to set in an economically optimal way due to political considerations inter alia. Focusing on uncertainty, Anderson et al. (2007) use a so-called market distribution function to explore the consequences of an interconnection, while Fleten et al. (2011) take a real options approach to determine optimal investment timing and sizing for a cable between Germany and Norway. A limitation of these papers is that they do not account for the behaviour of market participants in response to the interconnection addition. By contrast, Borenstein et al. (2000) use a game-theoretic approach to demonstrate the potential for enhanced competition from even modest transmission expansion in a two-node Cournot oligopoly model. Likewise, Sauma and Oren (2009) examine how financial transmission rights (FTRs) align electricity producers' incentives with socially desirable outcomes. Yet, the efficient outcomes hypothesised by Hogan (1992) under a so-called merchant investor are subverted if market power exists (Joskow and Tirole, 2005). In a tri-level model with transmission planning, generation expansion, and market operations, Sauma and Oren (2006) illustrate the complexity faced by a TSO in obtaining politically feasible transmission-expansion plans. However, these papers focus on economically efficient transmission expansion without consideration of environmental factors.

In the past decade, as environmental concerns have risen to the fore, the TSO's task has become more complex as it must anticipate RE penetration in an effort to provide the complementary transmission expansion. For example, it is legally binding for European Union (EU) member states to obtain $20 \%$ of their energy from RE resources by the year 2020 (European Commission, 2009), and California has an ambitious target for an $80 \%$ reduction in greenhouse gas (GHG) emissions relative to 1990 levels by the year 2050 (Executive Order B-30-15). As a consequence of this dramatic shift in the future energy landscape, the TSO is called upon to coordinate transmission investment with anticipated RE adoption by power companies, e.g., Statnett in Norway (Borgen, 2016) and FERC Order No. 1000 in requiring public utility transmission providers to consider policy measures in the cost effectiveness of their transmission planning (FERC, 2011). In effect, transmission capacity, along with curbs in consumption and greater reliance on $\mathrm{RE}$ resources, is to serve as another tool for environmental control (Vara, 2016). Yet, Hobbs (2012) cautions against the use of proxy

\footnotetext{
${ }^{1}$ TSOs are independent entities responsible for the bulk transmission of electric power on high-voltage electric networks. They also provide non-discriminatory grid access to other electricity market participants, viz., power companies, distributors, traders, and customers, and ensure the transmission network's safe operation. Finally, in many countries, TSOs are also charged with the development of the grid infrastructure, which includes "facilitating secure integration of new generation sources, particularly renewable energy, as well as significantly contributing to the EU's greenhouse gases reduction and renewable energy supply goals." (ENTSO-E, 2018)
} 
measures, e.g., those of the EU, because they may obfuscate economic tradeoffs. Carrying on in this spirit, bi-level models by Baringo and Conejo (2013) and Maurovich-Horvat et al. (2015) tackle transmission expansion with RE adoption in a market setting. The latter, in particular, demonstrates the somewhat unexpected results that may arise when power companies have market power: their withholding of capacity could actually incentivise TSOs to build transmission lines that connect RE-rich regions with load centres. Meanwhile, focusing on only electricity market operations without consideration of transmission investment, Downward (2010) uses an equilibrium problem with equilibrium constraints (EPEC) to indicate how congestion alleviation under a carbon charge may actually increase total emissions.

The environmental economics literature explicitly examines the efficiency of environmental policies in presence of pre-existing distortions, e.g., market power. In the electricity industry, evidence of post-deregulation market power has emerged, e.g., from the U.K. (Joskow, 2008). While growing penetration of RE exacerbates the problem of "missing money" (Rintamäki et al., 2016), market power continues to be a challenge. Recent empirical evidence points to the occasional exercise of market power by fossil-fuel plants, and the need for flexibility due to increasing RE penetration may actually give more leverage to flexible producers in limited hours of the year. ${ }^{2}$ However, in a stylised model (such as ours) with only a single representative time period, analyses typically tackle the extreme cases of perfect competition and Cournot oligopoly that may be encountered in contemporary electricity markets in order to provide policy insights. In this vein, Barnett (1980) demonstrates that in the extreme case of a monopoly, a pollution tax may worsen social welfare. Intuitively, under imperfect competition, there are two potential distortions: underproduction and pollution. Since a pollution tax based on marginal externality damages, e.g., a Pigouvian tax, aims to curb consumption by internalising externalities, it may cause imperfectly competitive firms to reduce their output further. Therefore, the resulting loss in consumer surplus from production withholding could more than offset the benefit from mitigation of pollution. Requate (2006) additionally notes that it would be optimal not only to reduce the pollution tax rate below that of the marginal damage from externality but also to set a negative tax to incentivise more production.

We investigate this interaction between economic efficiency and the cost of damage from emissions by explicitly considering the transmission line as an environmental instrument. Our intention

\footnotetext{
${ }^{2}$ In the PJM Interconnection, the market on aggregate is competitive, i.e., prices set by marginal offering units close to their marginal costs, but price markups did occur during high demand periods in the local market, i.e., created by binding transmission constraints. For example, in the PJM real-time market, price markups were $\$ 4.94 / \mathrm{MWh}$ on average during 2017, which is $16 \%$ of the load-weighted average price (PJM, 2018). Likewise, while the day-ahead market in California is generally competitive, concerns about the exercise of local market power due to congestion induced the incorporation of additional mitigation measures in the 5-minute market (CAISO, 2018). From Europe, an empirical analysis of Nord Pool during 2010-2013 finds evidence of local market power in Swedish pricing areas, which are dominated by hydropower (Tangerås and Mauritzen, 2018). The German balancing market is also empirically found to be susceptible to the exercise of market power based on an analysis during 2009-2011 (Just and Weber, 2015). Strategic manipulation with intermittent RE has occurred in California as a result of renewable portfolio standards with more ramping requirements. For example, firms exploit CAISO's rule that a generator will be paid to ramp down its output by under-bidding one day (so that it is dispatched) while simultaneously over-bidding at $\$ 999 / \mathrm{MWh}$ the next day so that the generator will be requested by CAISO to reduce its output (Fitzpatrick and Barrett, 2013).
} 
is to develop a framework for assessing the welfare implications of using transmission capacity as a tool to regulate emissions. Towards that end, we address the problem of a welfare-maximising TSO that internalises the cost of damage from carbon emissions in a deregulated electricity industry. We use a bi-level approach in which the TSO at the upper level anticipates the possibly strategic behaviour of power producers that make generation decisions at the lower level. We obtain closedform solutions to the transmission-expansion problem under three settings: central planning $(\mathrm{CP})$, perfect competition (PC), and Cournot oligopoly (CO). Vis-à-vis the environmental economics literature, viz., Barnett (1980) and Requate (2006), we first find that the transmission line in a decentralised electricity industry under PC can still be used as a tool for controlling the externality for a moderate cost of damage from emissions. In effect, the TSO reduces the transmission capacity to curb fossil-fuel generation in favour of $\mathrm{RE}$ output. As in the literature, increasing the carbon charge under PC aligns the result with the socially optimal solution as the transmission capacity is increased monotonically. By contrast, imposing such a carbon charge under the CO setting may actually worsen social welfare even though RE generation increases. This is mainly because the carbon charge suppresses already choked consumption, thereby further deteriorating social welfare. Interestingly, with a high damage cost parameter under PC, a less-than-full carbon charge imposed on industry actually leads to "too much" transmission capacity as the TSO attempts to mitigate the environmental cost by facilitating extra RE imports. Increasing the carbon charge to cover $100 \%$ of the cost of damage from emissions under PC finally aligns incentives perfectly and results in lower transmission capacity. Thus, at a relatively high level of the damage cost, the transmission capacity behaves non-monotonically with the carbon charge under PC even if maximised social welfare increases monotonically, which is a result that has previously not been studied in environmental economics and sheds light on how the carbon charge interacts with a physical transmission network under different market settings.

The rest of the paper is structured as follows. Section 2 lays out our modelling assumptions, while Section 3 solves the model under three market structures without a carbon charge. Next, Section 4 examines the impact of a carbon charge on market outcomes and social welfare. The main insights are illustrated via numerical examples in Section 5, while Section 6 summarises our contributions and offers directions for future research. All proofs of propositions are in the Appendix.

\section{Assumptions}

\section{Parameters}

$A_{j} \quad$ Intercept of the inverse demand function at node $j=F, R[\$ / \mathrm{MW}]$

$B_{j} \quad$ Slope of inverse demand function at node $j=F, R\left[\$ / \mathrm{MW}^{2}\right]$

$C_{j} \quad$ Levelised cost of power generation at node $j=F, R[\$ / \mathrm{MW}]$

$C_{T} \quad$ Levelised cost of transmission capacity $[\$ / \mathrm{MW}]$

D Rate of increase in marginal cost of greenhouse gas emissions $\left[\$ / \mathrm{MW}^{2}\right]$ 


$\begin{array}{ll}\text { Primal Variables } \\ k & \text { Transmission capacity [MW] } \\ t & \text { Net power imported by node } R \text { from node } F[\mathrm{MW}] \\ x_{j} & \text { Power consumption at node } j=F, R[\mathrm{MW}] \\ y_{j} & \text { Power generation at node } j=F, R[\mathrm{MW}]\end{array}$

We assume that there are two nodes denoted by $j=F, R$ in a power system that are initially disconnected. At each node, there is one profit-maximising power generator with linear levelised cost functions $c_{j}\left(y_{j}\right)=C_{j} y_{j}$ such that $C_{R}>C_{F}$ (in $\$ / \mathrm{MW}$ ) with power generator $R(F)$ representing $\mathrm{RE}$ (fossil fuel, FF). This means that (i) there is only FF (RE) generation at node $F$ (node $R$ ) and (ii) the levelised cost of power generation from $\mathrm{FF}$ is assumed to be less than that from RE. The inverse demand function at each node is affine in total consumption, i.e., $p_{j}\left(x_{j}\right)=A_{j}-B_{j} x_{j}$, where $A_{j}$ (in $\$ / \mathrm{MW}$ ) and $B_{j}$ (in $\$ / \mathrm{MW}^{2}$ ) are the intercept and slope parameters, respectively. Thus, the inverse demand function, denoted by $p_{j}\left(x_{j}\right)$, indicates the maximum price that consumers at node $j$ are willing to pay to consume an additional unit of power at a given amount, $x_{j} \in\left[0, A_{j} / B_{j}\right]$, of power consumption. Intuitively, the maximum price that consumers are willing to pay is decreasing with the amount of power consumed. The actual price paid for power at node $j$ is the equilibrium price, $p_{j}^{*}$, which results from the intersection between supply and demand, cf. page 63 of Bushnell et al. (2017). Since the final product, power, is identical at the point of delivery in a power pool, it is not possible for consumers to discern its origin, i.e., the product is assumed to be homogenous as consumers do not know if power is of FF or RE origin. A welfare-maximising TSO at the upper level builds a transmission line of capacity $k$ (in MW) between nodes $F$ and $R$ with levelised construction cost $C_{T}$ (in $\$ / \mathrm{MW}$ ). Since only the TSO is regulated, it alone faces the cost of damage from emissions, $\frac{1}{2} D y_{F}^{2}$, where $D \geq 0$ (in $\$ / \mathrm{MW}^{2}$ ) is the damage cost parameter and $y_{F}$ is the amount of power generated from FF. In environmental economics, the damage cost function's convexity is a standard assumption, e.g., Requate (2006) supposes that the damage cost function is twice continuously differentiable, increasing, and weakly convex with strict convexity imposed as required (even though pollution itself may be only proportional to output). ${ }^{3}$

At the lower level, each generator makes its production decision, $y_{j} \geq 0$ (in MW), while an independent system operator (ISO) that is distinct from the TSO clears the markets in order to maximise the change in social welfare, i.e., transmits power from node $F$ to node $R(t>0$, in MW) or vice versa $(t<0)$ while taking the generators' production decisions as given (Sauma and Oren, 2006). ${ }^{4}$ This pool-type power market is common in many regions worldwide. In power pools, electricity is literally "pooled" without any discrimination of quality (e.g., RE or FF) due to the

\footnotetext{
${ }^{3}$ As a consequence of a convex damage cost function, it is plausible that if carbon charges were imposed on producers, then several small plants may be preferred to a single large one.

${ }^{4}$ While it may be the case that price volatility, which we do not address directly, affects the provision of balancing services and, thus, power flows, recent work has shown that intraday price differences in Denmark and Germany drive price volatility (Rintamäki et al., 2017). If we had multiple time periods in our model, then we could capture these
} 
physical nature of electrons. In such pool power markets, social welfare is measured by the quantity demanded by consumers due to the fact that consumers cannot discern the product's origin, cf. Schweppe et al. (1988); Hogan (1992); Chao and Peck (1996). ${ }^{5}$ Thus, consumption at nodes $R$ and $F$ (in MW) is $x_{R}=y_{R}+t$ and $x_{F}=y_{F}-t$, respectively, where $t$ is the net power imported by node $R$ from node $F$. Additionally, we assume $B_{j}>0, A_{F}>A_{R}>C_{R}>C_{T}>C_{F}>0$, and $\frac{A_{F}}{B_{F}}>\frac{A_{R}}{B_{R}}$ to reflect that (i) node $F$ is the load centre $\left(A_{F}>A_{R}\right.$ and $\left.\frac{A_{F}}{B_{F}}>\frac{A_{R}}{B_{R}}\right)$, (ii) each node's demand may be locally satisfied $\left(A_{j}>C_{j}\right)$, (iii) $\mathrm{RE}$ is more expensive than FF $\left(C_{R}>C_{F}\right)$, and (iv) transmission capacity is not prohibitively expensive $\left(C_{R}>C_{T}>C_{F}\right)$. We model a representative time period without uncertainty in demand and RE output. ${ }^{6}$

We justify the assumption that $\mathrm{RE}$ is remote from the load centre by noting that RE resources are usually located in specific solar- or wind-rich areas. In Germany, the increased penetration of wind farms in the north of the country has posed a challenge for the existing transmission system, which was historically configured to take power generated from FF plants in the south (where most of the industry is concentrated) to the rest of the country (Kunz, 2013). Similar to the German situation are the cases of Japan and Texas (i.e., wind-rich northern areas vs. FF-dominated southern areas). For example, the Organization for Cross-regional Coordination of Transmission Operators, Japan (OCCTO) is now investigating optimal investment in a longitudinal transmission network between the northern area (wind-rich but less populated area) and the Tokyo metropolitan area (FF-dominated area) (OCCTO, 2017). Another example may be off-shore wind power that uses a long direct current (DC) transmission line connected to a demand centre. Likewise, wind resources in Texas are located in the sparsely populated northwestern part of the state whereas the main cities are in the southeast, thereby necessitating substantial new transmission investment (Martin, 2016). Given this framework, we explore the following three distinct market settings:

Central planning (CP) This benchmark setting has a central planner controlling all generation, transmission capacity, and power flow to maximise social welfare considering damage from emissions. This results in a single-level quadratic program (QP) formulated in Section 3.1 as $(1)-(4)$.

Perfect Competition (PC) Generation decisions at the lower level are made by price-taking $\mathrm{RE}$ and FF power sectors who take the transmission capacity and power flow as given along with the decisions of the other power sector and maximise their profits. For example, the FF producer

peak/off-peak effects. However, this omission is mitigated by the fact that we assume that RE generation is backed up by storage, which is increasingly needed in European electricity markets (von Hirschhausen, 2014).

${ }^{5}$ Consumers also have no incentive to pay to reduce emissions because the absence of emissions, i.e., "cleanliness," is a public good with positive externalities, which are impossible to be retained fully by any consumer. Thus, the dominant strategy of any consumer would be to purchase fewer units of cleanliness since it could free ride on the purchases of others, thereby leading to a prisoner's dilemma with no purchases of cleanliness. For this reason, the inverse demand function for consumers in most of the economics literature is modelled as being dependent only on power consumption and not on emissions at all (Requate, 2006; Bushnell et al., 2017). Instead, the externality stemming from emissions is captured by damage cost function that we include in the TSO's objective function.

${ }^{6}$ We avoid RE intermittency by assuming that the $\mathrm{RE}$ generator uses $\mathrm{RE}$ and storage. Consequently, RE with storage also means $C_{R}>C_{F}$, although the levelised cost of standalone RE is approaching parity with that of FF. 
takes the transmission capacity, $k$, power flow, $t$, and the RE producer's generation, $y_{R}$, as given when solving its profit-maximisation problem in line with the standard assumptions of a Nash game (Hobbs, 2001). The ISO determines the power flow, $t$, in order to maximise the change in social welfare while taking the transmission capacity, $k$, and the power sectors' decisions, $y_{F}$ and $y_{R}$, as given (Sauma and Oren, 2006). At the upper level, the TSO builds transmission capacity to maximise social welfare constrained by the lower-level problems. This bi-level program may be re-cast as a mathematical program with equilibrium constraints (MPEC) if each lower-level problem is convex and may, thus, be replaced by its Karush-Kuhn-Tucker (KKT) conditions (Gabriel et al., 2012). The resulting MPEC is formulated in Section 3.2 as (19).

Cournot Oligopoly (CO) This is the same as $\mathrm{PC}$ except that each power sector behaves $\grave{a}$ la Cournot, i.e., it is able to raise the electricity price above the levelised cost while assuming that the other sector's decision is unchanged, whereas the ISO remains as a price taker. The corresponding MPEC is formulated in Section 3.3 as (25).

Since we will examine three market structures, we denote $\bar{\cdot}, \hat{\cdot}$, and $*^{*}$ as the optimal values for decision variables in $\mathrm{CP}, \mathrm{PC}$, and $\mathrm{CO}$ settings, respectively.

\section{Mathematical Model and Analytical Results}

\subsection{Central Planning}

The central planner selects power generation at nodes $F$ and $R, y_{F}$ and $y_{R}$, respectively, transmission capacity of the line built between nodes $F$ and $R, k$, and net flow of power from node $F$ to node $R, t$, in order to maximise social welfare by solving the following QP problem:

$$
\begin{array}{cl}
\max _{y_{R} \geq 0, y_{F} \geq 0, t, k \geq 0} & {\left[A_{F}\left(y_{F}-t\right)-\frac{1}{2} B_{F}\left(y_{F}-t\right)^{2}\right]+\left[A_{R}\left(y_{R}+t\right)-\frac{1}{2} B_{R}\left(y_{R}+t\right)^{2}\right]} \\
& -\sum_{j} C_{j} y_{j}-C_{T} k-\frac{1}{2} D y_{F}^{2} \\
\text { s.t. } \quad & \lambda^{-}:-k \leq t \leq k: \lambda^{+} \\
& y_{R}+t \geq 0: \beta_{R} \\
& y_{F}-t \geq 0: \beta_{F}
\end{array}
$$

The objective function in (1) is a simplified expression comprising consumer surplus, producer surplus, merchandising surplus, cost of transmission construction, and cost of damage from emissions. The complete breakdown of the social welfare expression is as follows. First, the gross consumer benefit from consumption of $x_{F} \mathrm{MW}$ of power at node $F$ is $A_{F}\left(y_{F}-t\right)-\frac{1}{2} B_{F}\left(y_{F}-t\right)^{2}$ and reflects the total benefit from consumption of $x_{F}$ MW of power, i.e., it is equal to the area under the inverse demand function at node $F, A_{F} x_{F}-\frac{1}{2} B_{F} x_{F}^{2}$, capturing the valuation of consumption. ${ }^{7}$ From this gross

\footnotetext{
${ }^{7}$ The marginal value of gross consumer benefit is $A_{F}-B_{F} x_{F}$, i.e., the marginal willingness to pay for an extra unit of consumption, which is always non-negative because $x_{j} \in\left[0, A_{j} / B_{j}\right]$. Hence, the gross consumer benefit is increasing in $x_{j}$ but at a decreasing rate and will not be decreasing in $x_{j}$.
} 
consumer benefit, the total cost of purchasing $x_{F}$ MW of power at node $F, p_{F}^{*} x_{F}$, must be subtracted to yield net consumer benefit (or, consumer surplus) at node $F, A_{F}\left(y_{F}-t\right)-\frac{1}{2} B_{F}\left(y_{F}-t\right)^{2}-p_{F}^{*} x_{F}$. Likewise, consumer surplus at node $R$ is $A_{R}\left(y_{R}+t\right)-\frac{1}{2} B_{R}\left(y_{R}+t\right)^{2}-p_{R}^{*} x_{R}$, where $p_{j}^{*}$ is the equilibrium price at node $j$ that results from equilibrium consumption matching supply and demand, i.e., it is the price paid by consumers to consume $x_{j}$ MW of power at node $j$ and is the price received by producers at node $j$. Second, producer surplus at node $j$ is $p_{j}^{*} y_{j}-C_{j} y_{j}$, and, third, the ISO's merchandising surplus from transmission operations is $\left(p_{R}^{*}-p_{F}^{*}\right) t$. Thus, using $x_{R}=y_{R}+t$ and $x_{F}=y_{F}-t$, we add these expressions for the consumer surplus, producer surplus, and merchandising surplus in order to obtain the first three terms in (1):

$$
\left[A_{F}\left(y_{F}-t\right)-\frac{1}{2} B_{F}\left(y_{F}-t\right)^{2}\right]+\left[A_{R}\left(y_{R}+t\right)-\frac{1}{2} B_{R}\left(y_{R}+t\right)^{2}\right]-\sum_{j} C_{j} y_{j}
$$

Completing the calculation of social welfare, the $-C_{T} k-\frac{1}{2} D y_{F}^{2}$ terms in (1) correspond to the costs of transmission construction and damage from emissions, respectively. Inequalities (2) constrain the net import at each node by the size of the transmission line. Equations (3) and (4) ensure that consumption at each node is non-negative. Finally, the term before or after the colon next to each constraint is the corresponding dual variable and may be interpreted as the shadow price on that constraint. 8

The KKT conditions for problem (1)-(4) are as follows, where " $\perp$ " denotes complementarity, i.e., $0 \leq a \perp b \geq 0 \Leftrightarrow a \geq 0, b \geq 0, a b=0$, and "u.r.s." denotes unrestricted in sign:

$$
\begin{gathered}
0 \leq y_{F} \perp-\left[A_{F}-B_{F}\left(y_{F}-t\right)\right]+C_{F}-\beta_{F}+D y_{F} \geq 0 \\
0 \leq y_{R} \perp-\left[A_{R}-B_{R}\left(y_{R}+t\right)\right]+C_{R}-\beta_{R} \geq 0 \\
0 \leq k \perp C_{T}-\lambda^{+}-\lambda^{-} \geq 0 \\
-A_{F}+B_{F}\left(y_{F}-t\right)+A_{R}-B_{R}\left(y_{R}+t\right)-\lambda^{+}+\lambda^{-}+\beta_{R}-\beta_{F}=0 \text { with } t \text { u.r.s. } \\
0 \leq \lambda^{+} \perp k-t \geq 0 \\
0 \leq \lambda^{-} \perp k+t \geq 0 \\
0 \leq \beta_{R} \perp y_{R}+t \geq 0 \\
0 \leq \beta_{F} \perp y_{F}-t \geq 0
\end{gathered}
$$

\footnotetext{
${ }^{8}$ For example, if $t>-k$ and $t=k$ in $(2)$, then $\lambda^{-}=0$ and $\lambda^{+} \geq 0$ indicate that the marginal value of transmission capacity in the direction from node $F$ to node $R$ is $\lambda^{+}$. (2) may be re-interpreted as two constraints:

(i) $t \leq k$ with corresponding dual variable $\lambda^{+}$.

(ii) $t \geq-k$ with corresponding dual variable $\lambda^{-}$.

If (i) is binding, i.e., $t=k$, then it means that the flow on the transmission line from node $F$ to node $R$ is exactly the transmission capacity, $k$. Correspondingly, the dual variable, $\lambda^{+}$, is non-negative and reflects the marginal value of an additional MW of transmission capacity, i.e., the shadow price. At the same time, since (ii) is not binding when (i) is binding, i.e., $t>-k$, its dual variable, $\lambda^{-}$, is zero, i.e., there is no marginal value for an additional unit of transmission capacity to accommodate transmission flow from node $R$ to node $F$ (Nash and Sofer, 1995).
} 
Without loss of generality, we assume that consumption at each node ${ }^{9}$ is strictly positive, which implies that the dual variables in (11)-(12) are zero, i.e., $\beta_{R}=0$ and $\beta_{F}=0$. Moreover, upper and lower bounds on the transmission capacity and power generation would be more realistic. However, the resulting constraints would involve integer variables, which would make it impossible for us to use an analytical framework that relies on KKT conditions. As our objective is to analyse the tradeoff between environmental and economic imperatives as reflected by (1), we have implicit upper bounds on decision variables based on the marginal benefits and marginal costs, e.g., KKT condition (7) states that a strictly positive transmission capacity will result only if the shadow price on the line is sufficient to cover its levelised cost and KKT condition (8) dictates that power flow on the line will be driven by the nodal price difference. Since the latter cannot be infinity in our model due to an upper bound on the willingness to pay for power at each node, we have implicit upper bounds on capacities. Admittedly, lower bounds on capacity are not incorporated within our model, but we cannot explicitly include them due to (i) the analytical framework and (ii) our focus on economic and environmental criteria rather than engineering ones. Numerical implementations involving integer variables (Maurovich-Horvat et al., 2015) are possible, and a promising new approach for incorporating binary variables in equilibrium problems may be extended to incorporate transmission constraints in future work (Huppmann and Siddiqui, 2018).

In order to solve the system in (5)-(12), we consider four cases that arise as the value of $D$ is increased from zero with analytical results summarised in Table $1 .^{10}$

CP Case 1 Electricity is generated only at node $F$ and transmitted to node $R$.

CP Case 2 Electricity is generated at both nodes $R$ and $F$ with some power flow from $F$ to $R$.

CP Case 3 The two nodes are separated with no power flow. From (9)-(10), we obtain $\bar{\lambda}^{+}(k) \geq 0$ and $\bar{\lambda}^{-}(k) \geq 0$. KKT condition (7) indicates that $C_{T} \geq \bar{\lambda}^{+}(k)+\bar{\lambda}^{-}(k)$. For ease of comparison with results from the bi-level models in Sections 3.2 and 3.3 as in Table 1, we parameterise the $\mathrm{CP}$ solutions on $k$.

CP Case 4 Electricity is generated at both nodes, but it flows from $R$ to $F$.

Using the solutions ${ }^{11}$ to the $\mathrm{QP}$, we assess the impact of the damage cost parameter, $D$, on capacity decisions (Proposition 1) and the cost of damage from emissions (Proposition 2):

Proposition 1. Under a solution for $C P$, ceteris paribus increases in $D$ result in:

Case 1: $\frac{\partial \bar{k}}{\partial D}<0, \frac{\partial \bar{y}_{F}}{\partial D}<0$.

Case 2: $\frac{\partial \bar{k}}{\partial D}<0, \frac{\partial \bar{y}_{F}}{\partial D}<0, \frac{\partial \bar{y}_{R}}{\partial D}>0$.

\footnotetext{
${ }^{9}$ We implicitly use the definitions for consumption, $x_{R}=y_{R}+t$ and $x_{F}=y_{F}-t$, in (3)-(4) and (11)-(12).

${ }^{10} \mathrm{We}$ can show that the case in which $k>0, y_{R}>0, y_{F}=0$, and $t<0$ does not occur. At the optimum, $-\left[A_{F}-B_{F}\left(y_{F}-t\right)\right]+C_{F}+D y_{F}=C_{F}-C_{R}-C_{T}<0$ holds with regard to (5), which is not feasible.

${ }^{11}$ We let $\bar{y}_{j} \equiv \bar{y}_{j}(\bar{k}), j=F, R$.
} 


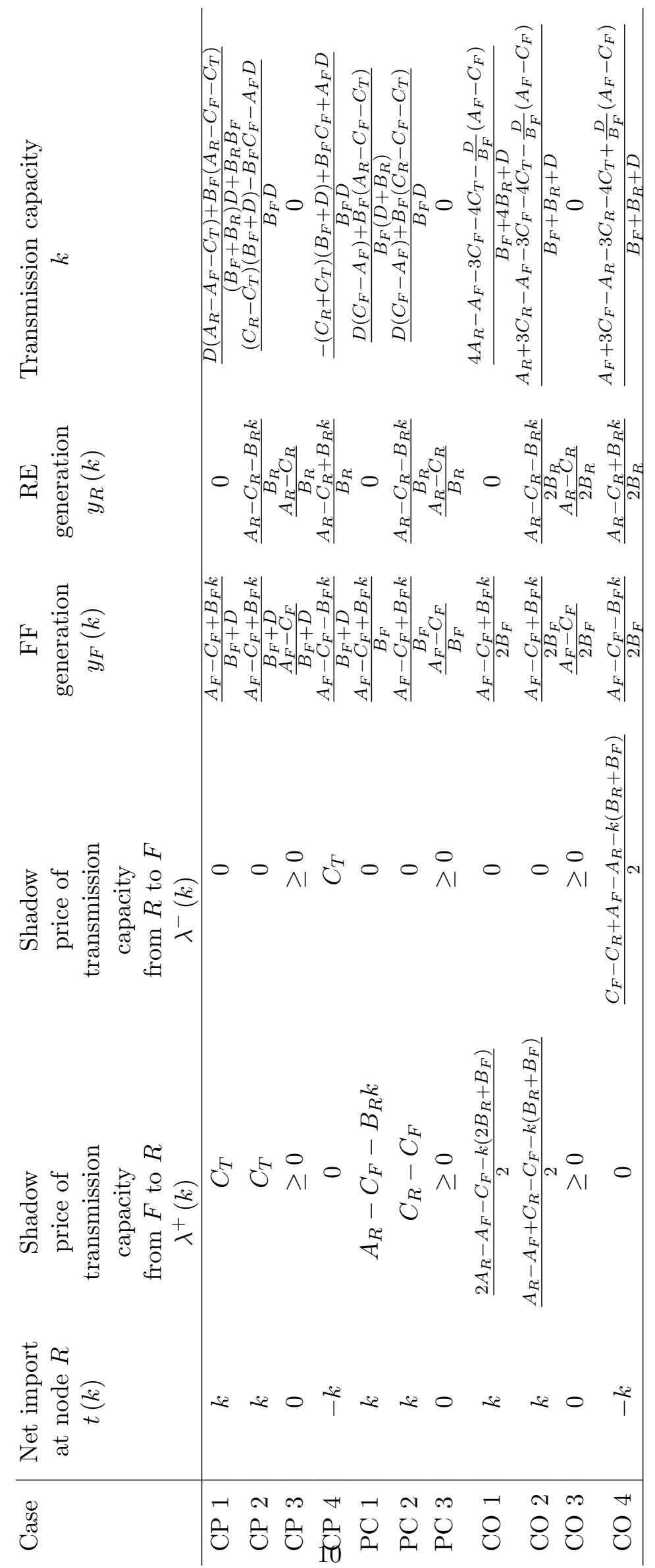

Table 1: Summary of analytical results 
Case 3: $\frac{\partial \bar{y}_{F}}{\partial D}<0, \frac{\partial \bar{y}_{R}}{\partial D}=0$.

Case 4: $\frac{\partial \bar{k}}{\partial D}>0, \frac{\partial \bar{y}_{F}}{\partial D}<0, \frac{\partial \bar{y}_{R}}{\partial D}>0$.

Proposition 2. Under a solution for $C P$, ceteris paribus increases in $D$ result in decreases in the damage cost from emissions in Cases 2 and 4.

Intuitively, for low $D$, all of the demand is satisfied via the cheapest generation source at node $F$ (Case 1). As $D$ increases, however, the levelised social cost of FF generation increases, and it is optimal to internalise the cost of damage from emissions by curbing consumption and, consequently, both production and transmission capacity, which is akin to a Pigouvian tax. Further increases in $D$ lead the levelised costs of RE and FF generation plus the cost of transmission capacity to be equated once the cost of damage from emissions is internalised (Case 2). Any marginal increase in $D$ within Case 2 will lead to subsequent reduction in FF generation and transmission capacity but not in consumption at node $R$ as RE generation will increase to compensate for the shortfall in net imports, thereby maintaining the price at node $R$ at the levelised cost of RE. For a high enough value of $D$, it may even be optimal not to build the line at all (Case 3 ) or to reverse the direction of prevalent transmission flows (Case 4). In the latter case, $\mathrm{RE}$ generation from node $R$ is sent to node $F$, which becomes a net importer. A marginal increase in $D$ leads to further displacement of FF generation by RE with a supporting increase in the transmission capacity. Finally, Proposition 2 indicates that the cost of damage from emissions decreases in Cases 2 and 4 only since the fraction of RE in the two-node system increases. By contrast, the results for Cases 1 and 3 are ambiguous, depending on the relation among $B_{R}, B_{F}$, and $D$.

\subsection{Perfect Competition}

In a deregulated setting, each producer maximises its own profit at the lower level by selecting the level of output while treating the transmission capacity set by the TSO at the upper level as a fixed quantity. In line with the standard definition of a Nash game (Hobbs, 2001), each producer also takes the generation decision of the other producer as given along with the ISO's decision about power flow, while the ISO takes the transmission capacity as fixed along with the generation decisions of both producers. The three independent single-objective problems at the lower level are as follows:

(i) The FF producer solves unconstrained problem (13) while taking $k, y_{R}$, and $t$ as given.

(ii) The RE producer solves unconstrained problem (14) while taking $k, y_{F}$, and $t$ as given.

(iii) The ISO solves problem (15) constrained by (2)-(4) while taking $k, y_{F}$, and $y_{R}$ as given.

Each producer acts as a price taker under perfect competition, i.e., the price at node $j, p_{j}$, is treated as a parameter.

$$
\begin{array}{ll}
\max _{y_{F} \geq 0} & p_{F} y_{F}-C_{F} y_{F} \\
\max _{y_{R} \geq 0} & p_{R} y_{R}-C_{R} y_{R}
\end{array}
$$


The ISO at the lower level manages power flow in order to maximise the change in social welfare (15) (Sauma and Oren, 2006).

$$
\max _{t} \quad\left[A_{R}-B_{R} y_{R}\right] t-\left[A_{F}-B_{F} y_{F}\right] t-\frac{1}{2}\left(B_{R}+B_{F}\right) t^{2}
$$

s.t. Equations (2)-(4)

Since each lower-level problem is convex, it may be replaced by its KKT conditions, which means that problems (13)-(15) become the following mixed complementarity problem (MCP):

$$
\begin{gathered}
0 \leq y_{F} \perp-\left[A_{F}-B_{F}\left(y_{F}-t\right)\right]+C_{F} \geq 0 \\
0 \leq y_{R} \perp-\left[A_{R}-B_{R}\left(y_{R}+t\right)\right]+C_{R} \geq 0 \\
-A_{R}+B_{R} y_{R}+A_{F}-B_{F} y_{F}+B_{R} t+B_{F} t+\lambda^{+}-\lambda^{-}-\beta_{R}+\beta_{F}=0 \text { with } t \text { u.r.s. }
\end{gathered}
$$

Equations (9)-(12)

Thus, the TSO's bi-level problem of choosing the optimal transmission capacity, $k$, in order to maximise social welfare while constrained by lower-level problems may be re-cast as the following MPEC:

$$
\begin{array}{cl}
\max _{\{k \geq 0\} \cup \Omega \cup \Xi} & \text { Equation (1) } \\
\text { s.t. } & \text { Equations (9)-(12), (16)-(17), (18) }
\end{array}
$$

where $\Omega \equiv\left\{\hat{y}_{R}(k) \geq 0, \hat{y}_{F}(k) \geq 0, \hat{t}(k)\right\}$ and $\Xi \equiv\left\{\hat{\lambda}^{+}(k) \geq 0, \hat{\lambda}^{-}(k) \geq 0, \hat{\beta}_{R}(k) \geq 0, \hat{\beta}_{F}(k) \geq 0\right\}$. We assume that $\hat{\beta}_{R}(k)=0$ and $\hat{\beta}_{F}(k)=0$ for the rest of analysis. Substitution of the solutions to the lower-level MCP allows the replacement of sets $\Omega$ and $\Xi$, thereby leading to the following non-linear programming (NLP) problem:

$$
\begin{array}{cc}
\max _{k \geq 0} \quad & A_{F}\left(\hat{y}_{F}(k)-\hat{t}(k)\right)-\frac{1}{2} B_{F}\left(\hat{y}_{F}(k)-\hat{t}(k)\right)^{2}-C_{F} \hat{y}_{F}(k)+A_{R}\left(\hat{y}_{R}(k)+\hat{t}(k)\right) \\
& -\frac{1}{2} B_{R}\left(\hat{y}_{R}(k)+\hat{t}(k)\right)^{2}-C_{R} \hat{y}_{R}(k)-C_{T} k-\frac{1}{2} D \hat{y}_{F}(k)^{2}
\end{array}
$$

As in Section 3.1, we have multiple solution $\operatorname{cases}^{12}$ depending on the value of $D$ :

PC Case 1 The lower-level solutions can now be substituted into NLP (20) to yield a QP problem parameterised on $k$. Using the fact that $\hat{y}_{F}^{\prime}(k)=1$, the first-order necessary condition (FONC) for the QP yields:

$$
-C_{F}-C_{T}-D \hat{y}_{F}(k)+A_{R}-B_{R} k=0
$$

\footnotetext{
${ }^{12}$ We can show that a possible Case 4 in which $\hat{k}>0, \hat{y}_{R}>0, \hat{y}_{F}>0$, and $\hat{t}<0$ is not feasible because $\hat{\lambda}^{-}=C_{F}-C_{R}<0$ holds from (18), which violates $\hat{\lambda}^{-} \geq 0$.
} 
Since (20) is a QP, the resulting solution is a global optimum, and, for $D=0$, the PC solution coincides with that of the central planner.

PC Case 2 Substitution of lower-level solutions into (20) again results in a QP. Using the facts that $\hat{y}_{F}^{\prime}(k)=1$ and $\hat{y}_{R}^{\prime}(k)=-1$, the FONC to (20) yields:

$$
-C_{F}+C_{R}-C_{T}-D \hat{y}_{F}(k)=0
$$

PC Case 3 The nodes are disconnected with $\hat{\lambda}^{+}(k) \geq 0$ and $\hat{\lambda}^{-}(k) \geq 0$ from (9)-(10).

Based on the solutions for the three cases, we can now perform comparative statics with respect to $D$ analogous to those in Propositions 1 and 2:

Proposition 3. Under a solution for PC, ceteris paribus increases in $D$ result in:

Case 1: $\frac{\partial \hat{k}}{\partial D}<0, \frac{\partial \hat{y}_{F}}{\partial D}<0$.

Case 2: $\frac{\partial \hat{k}}{\partial D}<0, \frac{\partial \hat{y}_{F}}{\partial D}<0, \frac{\partial \hat{y}_{R}}{\partial D}>0$.

Case 3: $\frac{\partial \hat{y}_{F}}{\partial D}=0, \frac{\partial \hat{y}_{R}}{\partial D}=0$.

Proposition 4. Under a solution for PC, ceteris paribus increases in $D$ result in a decrease and an increase in the damage cost from emissions in Cases 2 and 3, respectively.

Except for Case 3 with $\frac{\partial \hat{y}_{F}}{\partial D}=0$, the qualitative findings in Proposition 3 reflect those in Proposition 1 even though the optimal solutions are different. The reason for this difference is that the damage cost is not borne by producers, which means that a marginal increase in $D$ does not impact the producers' output. Unlike the CP setting, direct control over consumption is not possible under PC. Therefore, for Case 3, an increase in the damage cost parameter, $D$, leads to an increase in the damage cost (Proposition 4). However, the result for Case 1 is ambiguous, depending on the relation among $B_{R}, B_{F}$, and $D$.

\subsection{Cournot Oligopoly}

When generators behave as Cournot oligopolists at the lower level, they are able to raise prices above levelised costs. At the lower level, we have an MCP with each generator's KKT conditions for profit maximisation and the ISO's welfare-maximising net imports, viz., (9)-(12) and (18). To reflect market power, the generators' KKT conditions (16)-(17) are adjusted as follows:

$$
\begin{array}{cl}
\max _{y_{F} \geq 0} & {\left[A_{F}-B_{F}\left(y_{F}-t\right)\right] y_{F}-C_{F} y_{F}} \\
\Rightarrow & 0 \leq y_{F} \perp-\left[A_{F}-B_{F}\left(2 y_{F}-t\right)\right]+C_{F} \geq 0 \\
\max _{y_{R} \geq 0} & {\left[A_{R}-B_{R}\left(y_{R}+t\right)\right] y_{R}-C_{R} y_{R}} \\
\Rightarrow & 0 \leq y_{R} \perp-\left[A_{R}-B_{R}\left(2 y_{R}+t\right)\right]+C_{R} \geq 0
\end{array}
$$


As before, the lower-level problems may be replaced by their KKT conditions. Thus, the TSO's bi-level transmission-investment problem may again be rendered as an MPEC:

$$
\begin{array}{cl}
\max _{\{k \geq 0\} \cup \Omega \cup \Xi} & \text { Equation (1) } \\
\text { s.t. } & \text { Equations (9)-(12), (18), (23)-(24) }
\end{array}
$$

where $\Omega \equiv\left\{y_{R}^{*}(k) \geq 0, y_{F}^{*}(k) \geq 0, t^{*}(k)\right\}$ and $\Xi \equiv\left\{\lambda^{+, *}(k) \geq 0, \lambda^{-, *}(k) \geq 0, \beta_{R}^{*}(k) \geq 0, \beta_{F}^{*}(k) \geq 0\right\}$. Assuming $\beta_{R}^{*}(k)=0$ and $\beta_{F}^{*}(k)=0$, substitution of the solutions to the lower-level MCP allows the replacement of sets $\Omega$ and $\Xi$, thereby leading to the following NLP problem:

$$
\begin{array}{cl}
\max _{k \geq 0} \quad & A_{F}\left(y_{F}^{*}(k)-t^{*}(k)\right)-\frac{1}{2} B_{F}\left(y_{F}^{*}(k)-t^{*}(k)\right)^{2}-C_{F} y_{F}^{*}(k)+A_{R}\left(y_{R}^{*}(k)+t^{*}(k)\right) \\
& -\frac{1}{2} B_{R}\left(y_{R}^{*}(k)+t^{*}(k)\right)^{2}-C_{R} y_{R}^{*}(k)-C_{T} k-\frac{1}{2} D y_{F}^{*}(k)^{2}
\end{array}
$$

Variation of the $D$ parameter leads to four solution cases $^{13}$ as follows:

CO Case 1 Substitution of lower-level solutions and the fact that $y_{F}^{* \prime}(k)=\frac{1}{2}$ into (26) yields the FONC for the QP:

$$
-\frac{1}{2} A_{F}+\frac{1}{2} B_{F}\left(y_{F}^{*}(k)-k\right)-\frac{1}{2} C_{F}-C_{T}-\frac{1}{2} D y_{F}^{*}(k)+A_{R}-B_{R} k=0
$$

CO Case 2 Again, substitution of lower-level solutions into problem (26) leads to a QP. Using the facts that $y_{F}^{* \prime}(k)=\frac{1}{2}$ and $y_{R}^{* \prime}(k)=-\frac{1}{2}$, the FONC for the resulting QP yields:

$$
-\frac{A_{F}}{2}+\frac{B_{F}\left(y_{F}^{*}(k)-k\right)}{2}+\frac{A_{R}}{2}-\frac{B_{R}\left(y_{R}^{*}(k)+k\right)}{2}-\frac{C_{F}}{2}+\frac{C_{R}}{2}-C_{T}-\frac{D y_{F}^{*}(k)}{2}=0
$$

CO Case 3 Once the nodes are disconnected, $\lambda^{+, *}(k) \geq 0$ and $\lambda^{-, *}(k) \geq 0$ from (9)-(10).

CO Case 4 Substitution of lower-level solutions into problem (26) and using the facts that $y_{F}^{* \prime}(k)=-\frac{1}{2}$ and $y_{R}^{* \prime}(k)=\frac{1}{2}$, the FONC to the resulting QP yields:

$$
\frac{A_{F}}{2}-\frac{B_{F}\left(y_{F}^{*}(k)+k\right)}{2}-\frac{A_{R}}{2}+\frac{B_{R}\left(y_{R}^{*}(k)-k\right)}{2}+\frac{C_{F}}{2}-\frac{C_{R}}{2}-C_{T}+\frac{D y_{F}^{*}(k)}{2}=0
$$

Analogous to Propositions 3 and 4, we perform comparative statics with respect to $D$ :

Proposition 5. Under a solution for CO, ceteris paribus increases in $D$ result in:

Case 1: $\frac{\partial k^{*}}{\partial D}<0, \frac{\partial y_{F}^{*}}{\partial D}<0$.

Case 2: $\frac{\partial k^{*}}{\partial D}<0, \frac{\partial y_{F}^{*}}{\partial D}<0, \frac{\partial y_{R}^{*}}{\partial D}>0$.

\footnotetext{
${ }^{13}$ We can show that a potential Case 5 in which $k>0, y_{R}>0, y_{F}=0$, and $t<0$ does not occur. At the optimum, $-\left[A_{F}-B_{F}\left(2 y_{F}-t\right)\right]+C_{F}=\frac{B_{R}\left(C_{F}-A_{F}\right)+B_{F}\left[\left(C_{F}-A_{R}\right)+3\left(C_{F}-C_{R}\right)-4 C_{T}\right]}{4 B_{F}+B_{R}}<0$ holds with regard to (23). Hence, this case is not feasible.
} 
Case 3: $\frac{\partial y_{F}^{*}}{\partial D}=0, \frac{\partial y_{R}^{*}}{\partial D}=0$.

Case 4: $\frac{\partial k^{*}}{\partial D}>0, \frac{\partial y_{F}^{*}}{\partial D}<0, \frac{\partial y_{R}^{*}}{\partial D}>0$.

Proposition 6. Under a solution for CO, ceteris paribus increases in $D$ result in an increase in the damage cost from emissions in Case 3.

Although the solutions are different from the CP and PC cases, the comparative statics mirror the findings in the previous settings. Here, results for Cases 1, 2, and 4 are ambiguous, depending on the relation among $B_{R}, B_{F}$, and $D$. Finally, the damage cost parameter unambiguously decreases the maximised social welfare for all solutions:

Proposition 7. Under solutions for $C P, P C$, and $C O$, ceteris paribus increases in $D$ result in decreases in maximised social welfare.

\section{Impact of a Carbon Charge}

We now examine the effect of a carbon charge introduced in a deregulated industry, i.e., in the lower-level problem. Suppose that under the carbon charge system, the FF generator incurs the full or partial cost of damage from emissions, $\frac{1}{2} E D y_{F}^{2}$, where $0<E \leq 1$ is the carbon charge rate. The charge burden may be partial due to political reasons. If $E=1(E=0)$, then the $\mathrm{FF}$ generator incurs the entire damage cost (faces no environmental regulation as in the previous sections). Besides such a carbon charge, measures such as feed-in tariffs or renewable portfolio standards (RPS) may be employed to align private and social incentives. For example, Boomsma et al. (2012) take a real options approach to analysing the effects of these policy measures under uncertainty, while game-theoretic interactions with RPS either between firms (Nasiri and Zaccour, 2010; Tanaka and Chen, 2013) or between a policymaker and firms (Siddiqui et al., 2016) have also been the subject of recent investigation.

\subsection{Perfect Competition with a Carbon Charge}

At the lower level, the price-taking FF generator's KKT condition for profit maximisation is re-written as follows:

$$
\begin{array}{cl}
\max _{y_{F} \geq 0} & p_{F} y_{F}-C_{F} y_{F}-\frac{1}{2} E D y_{F}^{2} \\
\Rightarrow & 0 \leq y_{F} \perp-\left[A_{F}-B_{F}\left(y_{F}-t\right)\right]+C_{F}+E D y_{F} \geq 0
\end{array}
$$

As before, the lower-level problems may be replaced by their KKT conditions. Thus, the TSO's problem is again an MPEC to select the transmission capacity, $k$, in order to maximise social welfare:

$$
\begin{array}{cl}
\max _{\{k \geq 0\} \cup \Omega \cup \Xi} & \text { Equation (1) } \\
\text { s.t. } & \text { Equations (9)-(12), (17), (18), (30) }
\end{array}
$$

We can show that this problem can be solved as in Section 3.2 and yields the same first-best results as under $\mathrm{CP}$ if the $\mathrm{FF}$ generator bears full cost of damage from emissions. 
Proposition 8. If a full carbon charge, i.e., $E=1$, is imposed under PC, then the TSO's problem is equivalent to that under $C P$.

Therefore, a regulatory authority could use a full carbon charge to obtain the same outcome as in $\mathrm{CP}$, i.e., make the FF generator as concerned about emissions damage as a social planner. However, a full carbon charge is usually difficult to implement due to political reasons. With a partial carbon charge under $\mathrm{PC}$, i.e., $0<E<1$, the outcome deviates from that of $\mathrm{CP}$ and approaches the result of PC without any environmental regulation in Section 3.2. In a similar manner as in Section 3.2, it is possible to show that Cases 1 to 3 can occur, although we do not repeat the analysis here. We only note that Case 4 in which $\hat{k}>0, \hat{y}_{R}>0, \hat{y}_{F}>0$, and $\hat{t}<0$ can occur for $E>0$. This can be verified by deriving $\hat{\lambda}^{-}(k)=\frac{E^{2} D C_{T}+B_{F}\left[(1-E)\left(C_{F}-C_{R}\right)+E C_{T}\right]}{B_{F}+E^{2} D}$. Although $C_{F}-C_{R}$ is negative, $\hat{\lambda}^{-}(k)$ can be positive if $E$ is sufficiently large, thereby leading to Case 4 .

The subsequent analysis examines the impact of increasing the charge burden, i.e., raising $E$, on the transmission capacity. We focus on cases in which the optimal transmission capacity is positive.

Proposition 9. Under a solution for PC with $E \in(0,1)$, a more stringent carbon charge rate leads to an increase (a decrease) in the transmission capacity, i.e., $\frac{\partial \hat{k}}{\partial E}>0\left(\frac{\partial \hat{k}}{\partial E}<0\right)$, for Cases 1 and 2 (Case 4).

Proposition 9 suggests that the optimal transmission line under PC is smaller than (larger than) that under CP for Cases 1 and 2 (Case 4). Intuitively, consumption may be restricted under $\mathrm{CP}$ as $D$ increases in Cases 1 and 2 by internalising the cost of damage from emissions, thereby increasing prices. As a consequence, there is less need for transmission capacity to take power from node $F$ to node $R$. However, such recourse is unavailable to a TSO operating in a decentralised electricity industry. Instead, it responds optimally to higher $D$ in Cases 1 and 2 by directly reducing the transmission capacity to below the optimal level under CP. With the carbon charge, total consumption at node $R$ is not affected because the RE production cost effectively acts as a ceiling on the price. Thus, $\hat{y}_{R}+\hat{t}$ is unaffected, but increasing the carbon charge reduces $\hat{y}_{R}$ as more electricity is imported from node $F$. Conversely, at node $F$, increasing the carbon charge increases the marginal cost of production at node $F$, which causes consumption, $\hat{y}_{F}-\hat{t}$, to decrease. In effect, a higher charge frees up FF capacity to supply more of the demand at node $R$, which also increases $\hat{k}$. Meanwhile, in Case 4, the prevalent power flow has reversed as an exorbitant cost of damage from emissions has been imposed on the FF producer at node $F$. While a partial carbon charge curbs FF output at node $F$, it restricts neither it nor the consumption at node $F$ to the socially optimal level as under CP. This means that a larger transmission line is needed with a partial carbon charge under PC in comparison to $\mathrm{CP}$ in order to meet the excess quantity demanded at node $F$ via RE generation from node $R$. Hence, an increase in the carbon charge corrects for this externality, thereby causing the optimal transmission capacity to decrease to the CP level. 


\subsection{Cournot Oligopoly with a Carbon Charge}

At the lower level, the Cournot FF generator's KKT condition for profit maximisation is rewritten as follows:

$$
\begin{array}{cl}
\max _{y_{F} \geq 0} & {\left[A_{F}-B_{F}\left(y_{F}-t\right)\right] y_{F}-C_{F} y_{F}-\frac{1}{2} E D y_{F}^{2}} \\
\Rightarrow & 0 \leq y_{F} \perp-\left[A_{F}-B_{F}\left(2 y_{F}-t\right)\right]+C_{F}+E D y_{F} \geq 0
\end{array}
$$

The TSO's problem is again an MPEC to choose the transmission capacity, $k$, in order to maximise social welfare:

$$
\begin{array}{cl}
\max _{\{k \geq 0\} \cup \Omega \cup \Xi} & \text { Equation (1) } \\
\text { s.t. } & \text { Equations (9)-(12), (18), (24), (32) }
\end{array}
$$

In a similar way as in Section 3.3, we can show that Cases 1 to 4 can occur, although the analysis is not repeated here. We instead focus on the impact of the charge burden on the transmission capacity in cases with positive transmission capacities at the optimum.

Proposition 10. Under a solution for $C O$ with $E \in(0,1)$, if $E>1-\frac{B_{F}}{D}$, then a more stringent carbon charge rate leads to a decrease (an increase) in the transmission capacity, i.e., $\frac{\partial k^{*}}{\partial E}<0$ $\left(\frac{\partial k^{*}}{\partial E}>0\right)$, for Cases 1 and 2 (Case 4).

In order to understand the intuition for Proposition 10, first note that $E>1-\frac{B_{F}}{D}$ is equivalent to the condition $B_{F} y_{F}^{*}>(1-E) D y_{F}^{*}$. The left-hand (right-hand) side of the inequality refers to the impact on the social welfare from a marginal increase in production at node $F$ in terms of the reduced potential for market power (enhanced marginal cost of damage from emissions). Now, if $E$ were infinitesimally increased, then it would reduce production at node $F$, thereby leading to a marginal reduction in the cost of damage from emissions and a marginal increase in the potential for the exertion of market power. When $B_{F} y_{F}^{*}>(1-E) D y_{F}^{*}$, the gains from lower emissions as a result of a higher carbon charge are outweighed by the loss in welfare from the more potent effects of market power. Since maximised social welfare is bound to decrease from a higher carbon charge, it must be the case that $E>1-\frac{B_{F}}{D}$ corresponds to a situation in which the carbon charge is higher than the optimal level.

When power is transmitted from node $F$ to $R$ as in Cases 1 and 2, an infinitesimal increase in the carbon charge is undesirable as it will only lower maximised social welfare by curbing production at node $F$. Since node $F$ was already supplying consumers at node $R$, a further reduction in FF generation will be to the detriment of consumers at node $F$. As a countervailing measure, the TSO will optimally reduce the size of the transmission line in order to limit the damage to consumer surplus (and maximised social welfare overall) by restricting the power flow from node $F$ to $R$. By contrast, in Case 4, power is optimally transmitted from node $R$ to $F$ since the cost of damage from emissions is exceptionally high. Nevertheless, the carbon charge is already too high, and any further increases to it will lead to a further decrease in consumption at node $F$, which will lower 
social welfare as the loss in consumer surplus will more than offset the lower cost of damage from emissions. Again, as a countervailing measure, the TSO increases the capacity of the transmission line to bring more RE from node $R$ to $F$.

\subsection{Implications for Social Welfare}

In the environmental economics literature, it is known that the imposition of a Pigouvian tax may actually worsen social welfare in the presence of a pre-existing distortion, e.g., imperfect competition (Barnett, 1980; Requate, 2006). Since a producer with market power withholds production in order to raise its own profit, an emissions tax may actually be to the detriment of society even though it mitigates the externality. Thus, the literature concludes that the optimal emissions tax rate should be lower than the marginal damage when market power exists, i.e., $E<1$ in our setting. In fact, even a negative tax rate may be desirable when the alleviation from the withholding distortion outweighs the negative externality of emissions. However, this work typically does not consider network effects associated with the transmission line as we do here. Indeed, in the absence of direct control over production or taxation decisions, the TSO's only recourse is to vary the size of the line in balancing economic and environmental objectives.

We now formalise how the TSO uses the transmission line as an environmental control mechanism by demonstrating the impact of the charge rate, $E$, on social welfare. Note that since $E$ is a parameter and outside the control of the TSO, its only role is to determine the size of the transmission line. We let $s \hat{w}(E)$ and $s w^{*}(E)$ denote the maximised social welfare under PC and $\mathrm{CO}$, respectively, for a given rate of carbon charge.

Proposition 11. Under $P C$ with $E \in(0,1)$, a more stringent carbon charge rate leads to an increase in maximised social welfare, i.e., $\frac{\partial s \hat{w}}{\partial E}>0$.

Proposition 12. Under $C O$ with $E \in(0,1)$, if $E>1-\frac{B_{F}}{D}$, then a more stringent carbon charge rate leads to a decrease in maximised social welfare, i.e., $\frac{\partial s w^{*}}{\partial E}<0$. On the contrary, if $E<1-\frac{B_{F}}{D}$, then a more stringent carbon charge rate leads to an increase in maximised social welfare, i.e., $\frac{\partial s w^{*}}{\partial E}>0$.

The intuition for Proposition 11 is related to the findings of Propositions 8 and 9. Under PC without a carbon charge, there is over-generation from the RE plant at node $R$ as the cost of damage from emissions is not internalised. Consequently, high consumption at node $F$ prevents the export of relatively cheap energy to node $R$. For this reason, the optimal transmission line under PC without a carbon charge in Cases 1 and 2 is smaller than the socially optimal one under CP. Once the carbon charge is imposed and starts to increase from zero, consumers at node $F$ receive the price signal to reduce quantity demanded, which liberates relatively cheap FF generation at node $F$ for export to node $R$ with a concomitant increase (decrease) in transmission capacity (RE generation). In Case 4, a partial carbon charge does not curb consumption at node $F$ sufficiently even as FF generation is reduced. Thus, the shortfall between consumption and local generation needs to be made up via imports from $\mathrm{RE}$ generation at node $R$, which necessitates a larger transmission line than is 
socially optimal. Again, as the carbon charge increases, this distortion is corrected: consumption and $\mathrm{FF}$ generation at node $F$ are curbed along with both RE generation at node $R$ and transmission capacity. Hence, as the carbon charge rate approaches unity, the PC solution approaches the socially optimal one under CP.

If industry is imperfectly competitive, however, then simply setting $E=1$ will not suffice as indicated in Proposition 12. While this finding reflects results established in the environmental economics literature, it is, nevertheless, novel in that the transmission capacity also functions as an instrument for regulating emissions at node $F$, reducing consumption at node $F$, and increasing RE output at node $R$. The intuition here is related to the discussion of Proposition 10 as there are two consequences of increasing the carbon charge rate: (i) an enhanced potential for the FF producer at node $F$ to exert market power and (ii) a mitigation of the cost of damage from emissions. Since these two effects have opposing impacts on social welfare, the TSO is in a quandary. As a result, unlike in the PC case, simply setting the carbon charge rate to unity to cover full damage may actually worsen maximised social welfare if effect (i) more than offsets (ii), which is precisely the

situation captured by $E>1-\frac{B_{F}}{D}$. Therefore, although the TSO optimally adjusts transmission capacity in response to a marginal increase in an already high carbon charge rate, e.g., reducing (increasing) capacity in Cases 1 and 2 (Case 4) to limit the extent of the consumer surplus loss at node $F$, maximised social welfare decreases. By contrast, if the carbon charge rate is relatively low, i.e., the marginal impact of the cost of damage from emissions outweighs the potential welfare loss from an enhanced capability to exercise market power, then a higher carbon charge will improve social welfare.

We, therefore, summarise this section with the following results:

Proposition 13. Under PC, a full carbon charge rate, $E=1$, is optimal.

Proposition 14. Under $C O$, the optimal carbon charge rate is strictly less than one. If $B_{F}<D$ $\left(B_{F} \geq D\right)$, then the optimal charge rate is $E=1-\frac{B_{F}}{D}(E=0)$.

We emphasise here that we actually solve for the optimal carbon charge rate in each market setting in Propositions 13-14. In reality, regulators similarly tailor the carbon policy taking market conditions into account. For example, the competitiveness of EU industry and the threat of carbon leakage are reasons why EU ETS permits were grandfathered, i.e., distributed without charge (European Commission, 2018).

\section{Numerical Examples}

In this section, we illustrate the key analytical findings using parameter values indicated in Table 2. Furthermore, we also investigate the impact of the cost of damage from emissions by allowing for $D \in[0,0.5]$ and a carbon charge by allowing for $E \in[0,1]$.

\subsection{Results without a Carbon Charge}

For a relatively low damage cost and no carbon charge, Figure 1 demonstrates that the CP and PC solutions are similar: power is produced only at node $F$ and transmitted to node $R$, which 
Table 2: Data for numerical examples

\begin{tabular}{ll}
\hline Parameter & Value \\
\hline$A_{F}$ & 400 \\
$A_{R}$ & 200 \\
$B_{F}$ & 1 \\
$B_{R}$ & 1 \\
$C_{F}$ & 20 \\
$C_{R}$ & 80 \\
$C_{T}$ & 25 \\
\hline
\end{tabular}

corresponds to Case 1. However, apart from $D=0$, the two solutions are not identical. Indeed, $\mathrm{CP}$ leads to a higher power price at node $F$ than does PC. Meanwhile, the price at node $R$ under $\mathrm{CP}$ is exactly $\bar{p}_{F}+C_{T}$, i.e., $\$ 25 / \mathrm{MW}$ higher than the price at node $F$ (Equation (8)). In effect, CP curbs consumption at node $F$ such that the marginal social cost equals the marginal benefit from consumption, i.e., $\bar{p}_{F}=C_{F}+D \bar{y}_{F}$ (Equation (5)), thereby freeing up resources to meet demand at node $R$. As $D$ increases, both consumption and $\mathrm{FF}$ generation at node $F$ are curbed further along with the capacity of the transmission line. By contrast, the PC setting is unable to internalise the cost of damage from emissions without a carbon charge, i.e., $\hat{p}_{F}=C_{F}$ (Equation (16)), and leads to "too much" consumption at node $F$ (Siddiqui et al., 2016). Therefore, the TSO's only recourse under PC is to use the transmission line as an instrument for regulating emissions by investing in less capacity. While such a response is clearly sub-optimal from a welfare perspective as it leads to a higher cost of damage from emissions, it may be thought of as a second-best solution.

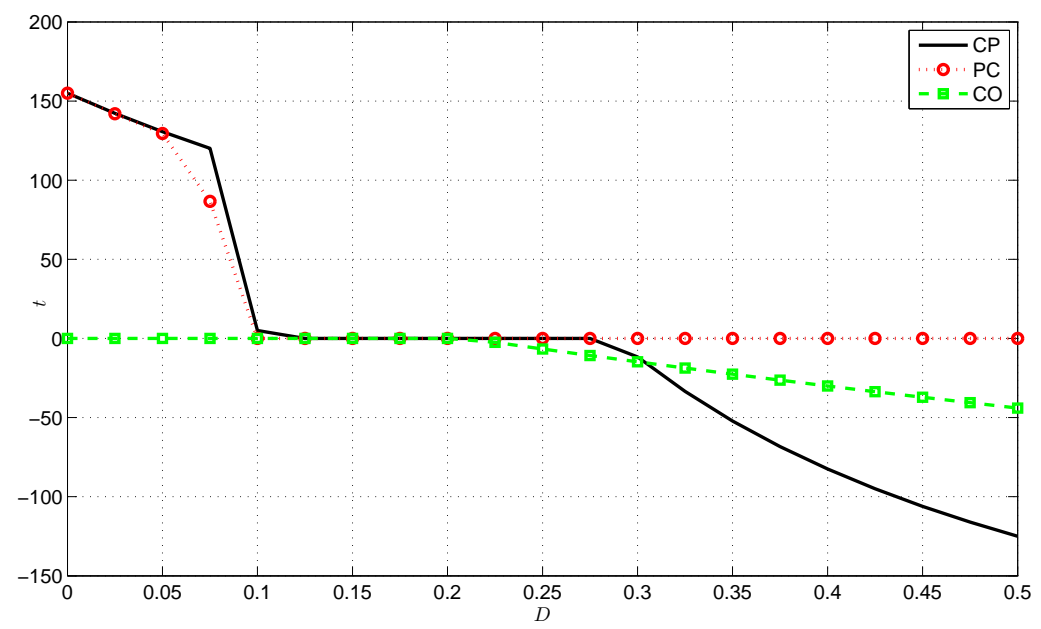

Figure 1: Optimal net import at node $R$ with respect to $D$

The contrast between the $\mathrm{CP}$ and PC settings becomes starker as $D$ increases to 0.075 . Here, $\mathrm{CP}$ continues to rely on FF generation at node $F$ (albeit in steadily decreasing quantities), whereas $\mathrm{PC}$ sees production from the RE generator at node $R$ (Case 2). The rationale for the switch in the 
PC setting is that the cost of damage from emissions is high enough to induce the TSO to decrease drastically the capacity of the transmission line. This intervention creates scarcity at node $R$ as the economic cost of producing power at node $F$ and transmitting it to node $R$, i.e., $\hat{p}_{F}+\hat{\lambda}^{+}$(Equation (18)), increases to exactly $C_{R}$ and enables RE generation to enter the market in response. Since the PC setting could not free up FF generation to supply node $R$ by curbing consumption at node $F$, it has to rely on RE generation. Under $\mathrm{CP}$, such parity between the two costs does not occur until $D=0.10$ when $\bar{p}_{F}+C_{T}$ is exactly $\$ 80 / \mathrm{MW}$. At this point, the CP setting produces nearly a quarter of the total power from RE sources at node $R$ and builds only a $5 \mathrm{MW}$ line (Figure 1). Indeed, only when the marginal social cost of the FF generator is high enough is it optimal to start relying on $\mathrm{RE}$ generation.

Figure 2 illustrates the producers' incentives at each node under PC. Note that under CP, the optimal transmission capacity is such that the marginal benefit and marginal cost of expansion are equated: the former stems from the reduction in RE production $\left(C_{R}\right)$, whereas the latter is due to the increase in FF production $\left(C_{F}\right)$, the increase in the transmission capacity $\left(C_{T}\right)$, and increase in the marginal cost of emissions damage $\left(D \bar{y}_{F}(k)\right)$. Although FF generation does not change like-forlike with transmission expansion, the resulting curb in consumption frees up precisely enough $\mathrm{FF}$ production to transmit to node $R$ to use the added transmission capacity. Since industry cannot internalise the cost of damage from emissions under the PC setting, the optimal FF generation is obtained by simply equating the marginal production cost with the shifted inverse demand curve at node $F$ (see Figure $2 \mathrm{~b}$ ). A ceteris paribus increase in $D$ curbs neither FF generation nor consumption at node $F$ for a given $k$, which prompts the TSO to reduce the size of the transmission line drastically. Although consumption at node $F$ is unaffected by the reduced transmission capacity, the lower net import at node $R$ reduces FF generation. Consequently, there is a like-for-like increase in $\mathrm{RE}$ generation at node $R$ to replace the curbed FF production (see Figure 2a). Under PC, the optimal transmission capacity is again determined by setting the marginal benefit of expansion to its marginal cost, i.e., $C_{R}=C_{T}+C_{F}+D \hat{y}_{F}(k)$. Since it is impossible for the TSO to curb consumption at node $F$, an infinitesimally larger transmission line leads to a like-for-like increase in FF output to transmit to node $R$. Hence, the optimal transmission capacity for a given $D$ is smaller under PC due to its higher marginal cost of expansion, i.e., $D \hat{y}_{F}(k)>D \bar{y}_{F}(k)$.

From Case 2, sustained increases in $D$ will make it more expensive to produce power from FF generation at node $F$ and to transmit it to node $R$. Once $\bar{p}_{F}+C_{T}>C_{R}$ under CP (and $\hat{p}_{F}+\hat{\lambda}^{+}>C_{R}$ under PC), it is actually more efficient to reduce planned transmission capacity, thereby leading to Case 3. Such autarky persists under CP until the cost of damage from emissions is high enough to warrant building a transmission line to send $\mathrm{RE}$ generation to node $F$, i.e., $\bar{p}_{F}=C_{R}+C_{T}$ (Case 4). However, this reversal of the prevalent power flow does not happen under $\mathrm{PC}$ as the price at node $F$ is too low to entice the RE generator as we showed in Section 3.2 (see Figure 1). Since the CP setting allows for the internalisation of the cost of damage from emissions, once $\mathrm{FF}$ generation is curbed to the extent that the price at node $F$ is $\$ 25 / \mathrm{MW}$ higher than that at node $R$, transmission capacity expansion is planned. The optimal size of the transmission line is 


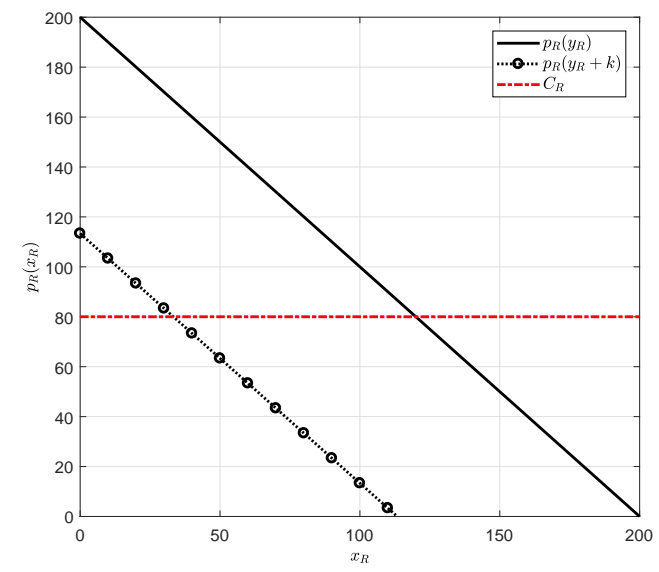

(a) Node $R$

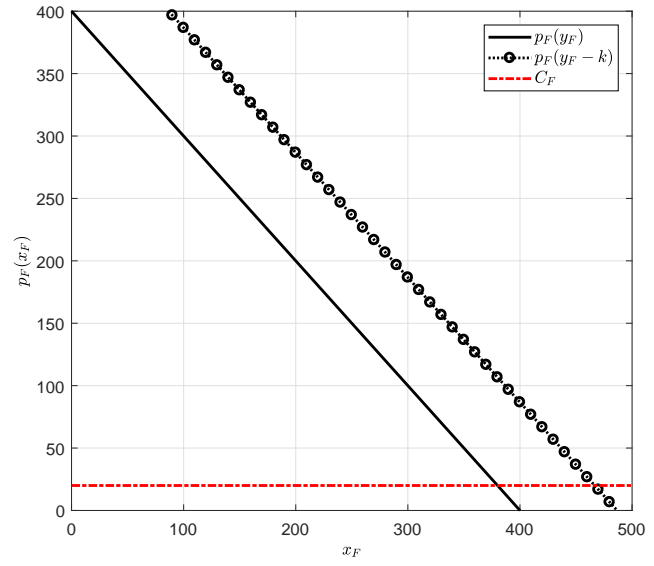

(b) Node $F$

Figure 2: Power sectors' incentives without a carbon charge under PC (Case 2)

determined by setting the marginal benefit of expansion (reduction in both the FF production cost and marginal cost of damage from emissions, $\left.C_{F}+D \bar{y}_{F}(k)\right)$ to its marginal cost (due to increased $\mathrm{RE}$ output and construction of the line, $\left.C_{R}+C_{T}\right)$. Consequently, an infinitesimal increase in $k$ leads to a like-for-like increase in RE output without affecting consumption at node $R$ and a less than like-for-like reduction (increase) in FF output (consumption at node $F$ ). For a given $k$, a ceteris paribus increase in $D$ will curb FF generation and, thus, consumption at node $F$. In order to prevent an inefficiently large price increase at node $F$, transmission capacity will be increased. Hence, RE generation will increase without affecting consumption at node $R$ as the surplus power will be transmitted to node $F$ to replace the curbed $\mathrm{FF}$ generation without affecting consumption there.

As Figure 1 indicates, Case 4 can occur under CO even without a carbon charge for sufficiently high $D$. The intuition for its observation is summarised by Figure 3 in which the marginal production costs are equated to the marginal revenues of the producers at each node. Thus, the equilibrium price always exceeds the marginal production cost. Since node $F$ has higher local demand than node $R$, it will have a higher equilibrium price due to the exercise of market power by the FF generator. Consequently, alleviation of this inefficient curb on consumption via a larger transmission line will enter into the TSO's calculus. From the perspective of the TSO, the optimal size of the transmission line depends on the marginal benefit of expansion (reduction in not only FF production and damage costs, $\frac{C_{F}}{2}+\frac{D y_{F}^{*}(k)}{2}$, but also choke on consumption, $\frac{p_{F}^{*}}{2}$ ) and its marginal cost (comprising not only the cost of transmission and RE production, $C_{T}+\frac{C_{R}}{2}$, but also loss in consumption at node $R, \frac{p_{R}^{*}}{2}$ ). In effect, as $D$ increases, the TSO will expand the transmission line to mitigate the cost of damage from emissions, which, in turn, alleviates the exertion of market power suppressing consumption at node $F$. These twin consequences of transmission expansion also enable the TSO to attain a higher maximised social welfare under CO than under PC (Figure 4). 


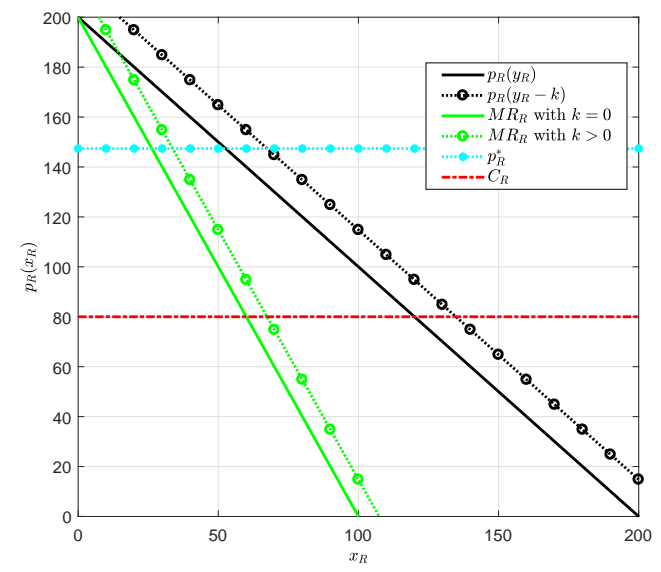

(a) Node $R$

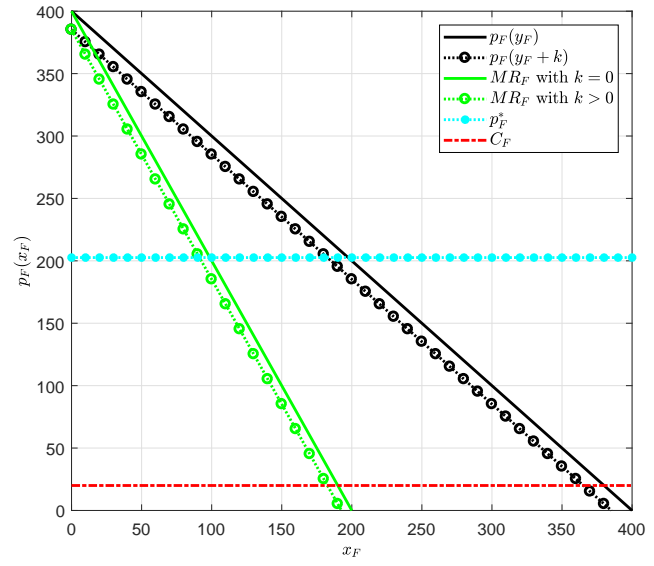

(b) Node $F$

Figure 3: Power sectors' incentives without a carbon charge under CO (Case 4)

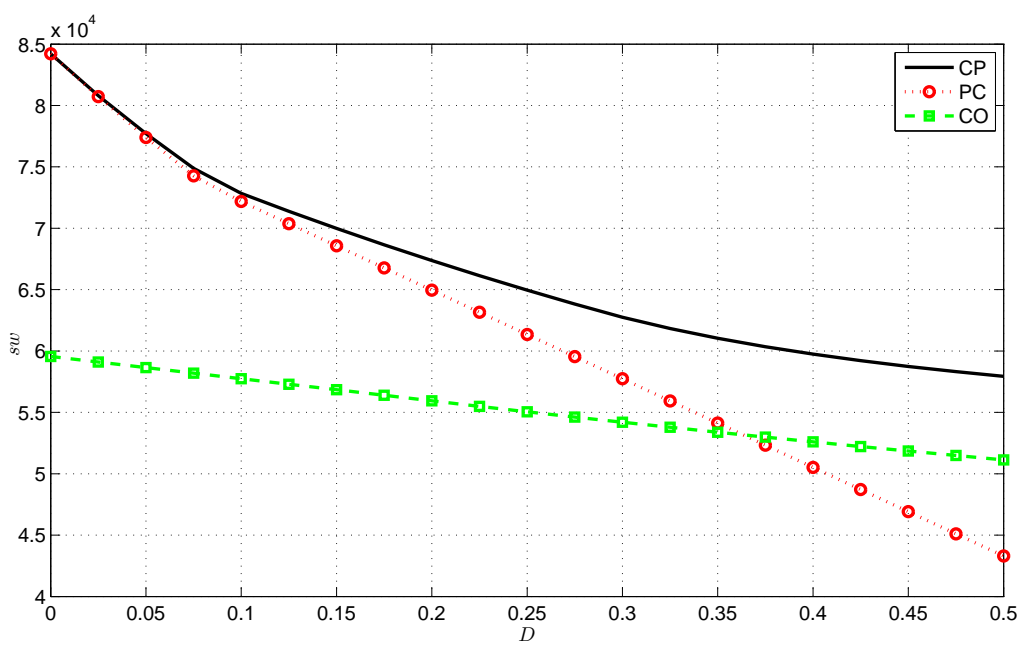

Figure 4: Maximised social welfare with respect to $D$

\subsection{Results with a Carbon Charge}

With a carbon charge, e.g., $E=0.50$ in Case 2 , total consumption at node $R$ under PC is not affected because the $\mathrm{RE}$ production cost is an upper limit on the price. Thus, $\hat{y}_{R}(k)+k$ is unchanged, but increasing the carbon charge reduces RE output, $\hat{y}_{R}(k)$, as more electricity is imported from node $F$ (see Figure 5a). Conversely, at node $F$, increasing the carbon charge increases the marginal cost of production at node $F$, which causes consumption, $\hat{y}_{F}(k)-k$, to decrease. In effect, a higher charge enables the TSO to free up FF capacity to supply more of the demand at node $R$, which also increases $k$ (see Figure 5b). As illustrated in Figure 6a, the optimal transmission line capacity increases with the carbon charge because the TSO's marginal cost of capacity expansion decreases as an infinitesimal increase in $k$ would lead to a less than proportional increase in FF output due to curbing of consumption at node $F$ (see Proposition 9). In Case 4 under PC, the 
transmission capacity actually decreases as $E$ increases from 0.75 to 1.00 , since a carbon charge of $E=0.75$ is insufficiently high to render a socially optimal curb in FF production. As partial compensation for this distortion, the TSO invests in "too much" transmission capacity for $E=0.75$ in order to incentivise a countervailing import of RE production at node $F$. When the carbon charge fully aligns private and social incentives, the TSO is able to reduce its investment, thereby leading to the finding that the transmission capacity behaves non-monotonically in $E$ for a high damage cost parameter under PC (see Proposition 9). Since a full carbon charge is able to align private incentives with social ones (Proposition 13), social welfare increases monotonically as the economically inefficient RE overproduction at node $R$ is curtailed without affecting consumption at node $R$ (see Proposition 11). Hence, even though there is the cost of increasing transmission capacity, lower consumption at node $F$, and slightly higher emissions, society is better overall (see Figure 6b).

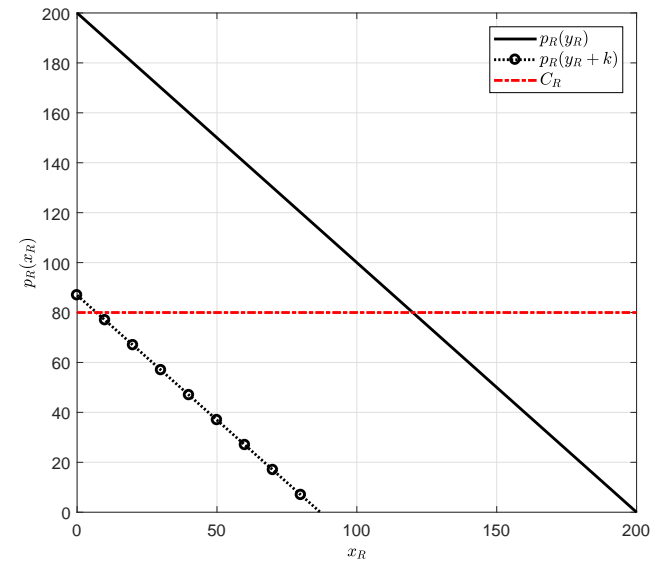

(a) Node $R$

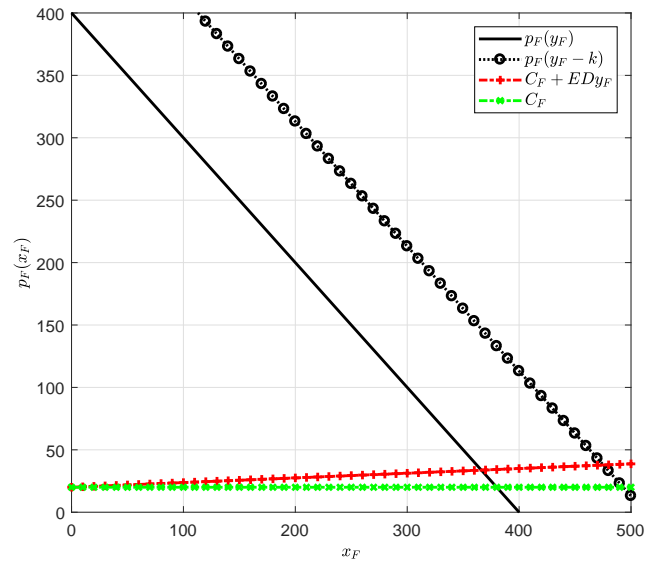

(b) Node $F$

Figure 5: Power sectors' incentives with a $E=0.50$ carbon charge rate under PC (Case 2)

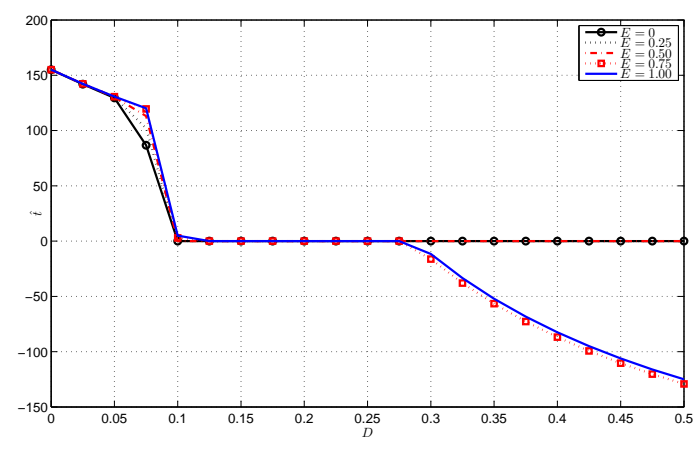

(a) Optimal net import at node $R$ with respect to $D$

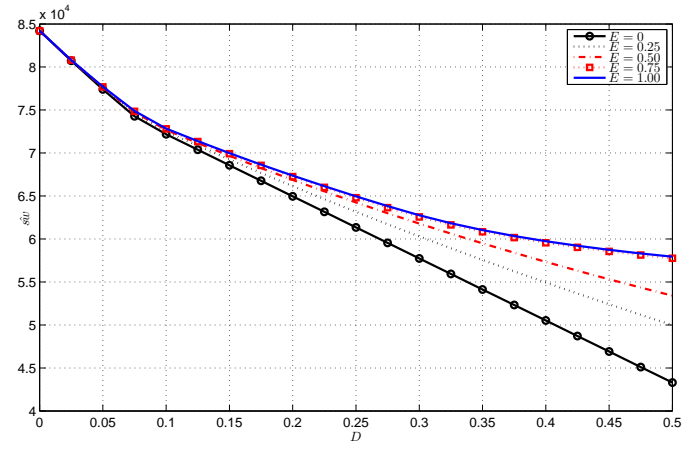

(b) Maximised social welfare with respect to $D$

Figure 6: Outcomes under PC setting with a carbon charge

In assessing the impact of a $E=0.50$ carbon charge rate in the $\mathrm{CO}$ setting, we focus on Case 
4. As in a situation without a carbon charge, we determine optimal RE production by setting net marginal revenue at node $R$ equal to the $\mathrm{RE}$ production cost. Increasing the carbon charge will cause $\mathrm{RE}$ generation to increase along with the price at node $R$, but the total consumption at node $R$ will decrease as more electricity is sent to node $F$ (see Figure 7a). Intuitively, by internalising the externality from emissions, consumers at node $F$ obtain the signal to reduce consumption and to pay a higher price (see Figure 7b). This incentivises more RE production, which is reinforced by the TSO's increasing transmission capacity to send more electricity to node $F$. As in Section 5.1, the TSO compares the marginal benefit of an infinitesimal increase in $k$ with its marginal cost. Although the latter is still equal to the cost of augmenting transmission and RE production, $C_{T}+\frac{C_{R}}{2}$, plus the value of lost consumption at node $R, \frac{p_{R}^{*}}{2}$, the marginal benefit is higher due to the imposition of a carbon charge. The marginal benefit from increasing $k$ is related to two factors: increased consumption at node $F$ and less FF generation. First, the marginal value of alleviating the choke on consumption via an infinitesimally larger transmission line increases with the carbon charge because consumption at node $F$ was already low from internalising the cost of damage from emissions. Second, the marginal benefit from reduction in FF production and damage costs via an infinitesimally larger transmission line decreases with the carbon charge because there is less FF output to mitigate. Since the increase in the marginal benefit from higher consumption at node $F$ outweighs the decrease in marginal benefit from reduction in FF output, the marginal benefit of expanding transmission capacity increases overall in the presence of the carbon charge. Therefore, $k$ increases with $E$ as shown in Proposition 10 and illustrated by Figure 8a for Case 4. Although the TSO's best response to the imposition of a carbon charge is to increase the transmission capacity, maximised social welfare is actually lower than without a charge as long as $E>1-\frac{B_{F}}{D}$ (see Figure 8b). As mentioned in the discussion of Proposition 12, the CO setting presents two opposing challenges for the TSO to address: the exertion of market power (especially at node $F$ ) reflected by $B_{F}$ and the cost of damage from emissions indicated by $D$. If the former is relatively large, then the use of a carbon charge will merely enhance the scope for the exercise of market power. In this instance, since $B_{F}>D$, the optimal carbon charge should be zero, i.e., $E=0$, according to Proposition 14. 


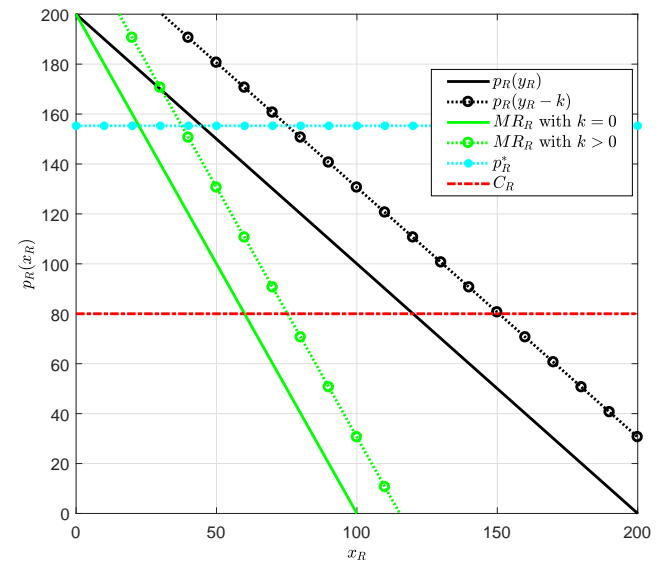

(a) Node $R$

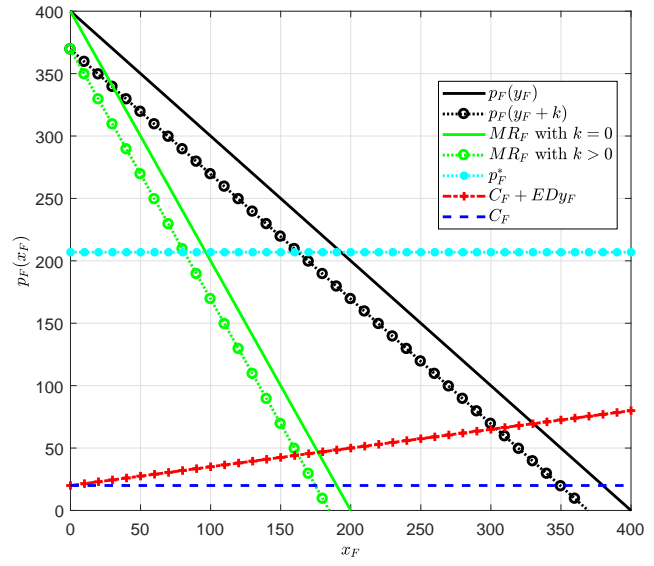

(b) Node $F$

Figure 7: Power sectors' incentives with a $E=0.50$ carbon charge rate under CO (Case 4)

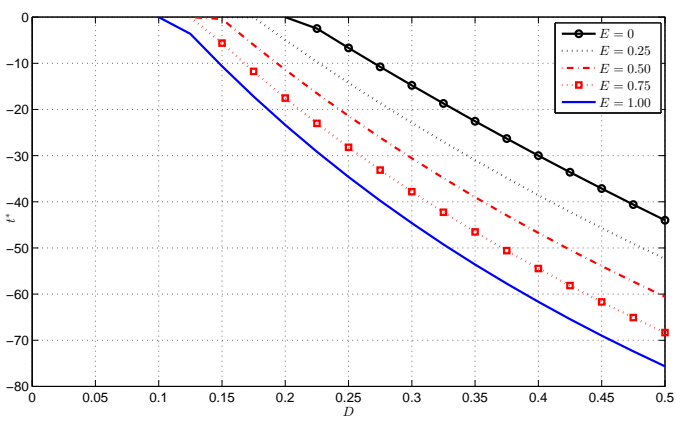

(a) Optimal net import at node $R$ with respect to $D$

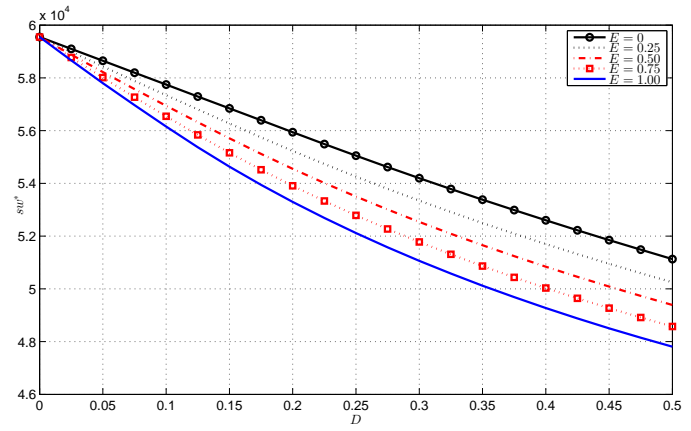

(b) Maximised social welfare with respect to $D$

Figure 8: Outcomes under $\mathrm{CO}$ setting with a carbon charge

\section{Conclusions}

The proliferation of RE resources as a means to mitigate climate change will typically require an augmentation of the transmission network. Consequently, expanding transmission capacity in order to balance economic and environmental objectives poses an opportunity as well as a significant challenge for a regulated TSO. In particular, a TSO must anticipate the response of profit-maximising power companies that invest in generation capacity when making its socially optimal transmission expansion. While the literature has addressed such anticipative transmission-expansion problems, research is still limited on the implications of using the transmission line as an instrument for regulating an environmental externality, which is likely to play an increasing role with ambitious climate targets set forth by various countries and regions.

In parallel with the environmental economics literature, we show how curbs on consumption, greater reliance on RE resources, and enhanced transmission capacity are all intertwined measures for limiting the cost of damage from emissions. However, it is only in the idealised CP setting 
that the socially optimal balance among the three is attained. Indeed, in a deregulated industry, the TSO can only indirectly influence consumption and production decisions through its sizing of the transmission line. We explore this dilemma via a bi-level modelling framework that we solve analytically in both PC and CO settings. Our main findings are that the solution under PC inhibits the TSO's ability to incentivise a curb in consumption in line with the cost of damage from emissions. As a countervailing measure, the TSO constructs a smaller transmission line to limit production from the FF generator and relies to a greater extent on RE output. Thus, although this result appears to be in line with certain policy targets, e.g., the EU's 20-20-20 energy and climate package, it is actually economically inefficient because there is "too much" RE generation instead of less consumption. Next, under a $\mathrm{CO}$ setting, the exercise of market power by producers provides the impetus for a reversal of the prevalent power flow as the node with RE generation becomes a net exporter provided that the damage cost parameter is high enough. Somewhat counterintuitively, a CO setting provides greater scope for the TSO to maintain social welfare as it is able to use the transmission capacity to alleviate both economic and environmental distortions by routing more $\mathrm{RE}$ output. Therefore, for an extremely high damage cost parameter, the maximised social welfare under $\mathrm{CO}$ is even higher than under $\mathrm{PC}$.

In order to correct for such externalities, it is appealing to deploy a carbon charge on FF generation. Under PC, we prove that such a measure results in perfect alignment of incentives: an increase in the carbon charge while holding the transmission capacity fixed reduces FF generation and lowers consumption enough to liberate FF capacity to be used in place of socially inefficient RE output. Recognising this change in incentives, the TSO increases the transmission capacity to the socially optimal level as in CP, but the transmission capacity under PC does not increase monotonically with the carbon charge for a high damage cost parameter as the TSO may overcompensate for the insufficient curb in FF production by facilitating more RE imports at node $F$. Moreover, a carbon charge under CO may worsen outcomes if consumption is already restricted by the exercise of market power. Indeed, while the carbon charge leads the TSO to increase the transmission capacity to supplant FF generation by RE output, the resulting loss in consumer surplus at both nodes leads to a net reduction in social welfare. Although this undesirable effect of a carbon charge in a setting with market power is known in the environmental economics literature, we have demonstrated that it may also arise when there is recourse to a transmission line as a mitigation measure. Therefore, proxies for climate control should be taken with caution, especially in the presence of market power.

For future work, the model could be expanded to handle more realistic features such as uncertainty in RE output and demand. Due to the complexity and range of strategies available to market players in such a setting, a numerical solution approach may be taken. On the other hand, allowing for distinct generation-expansion and operational decisions would lead to a tri-level model that would be interesting to solve analytically (Murphy and Smeers, 2010). Finally, informational asymmetry stemming from quality differentiation between FF and RE by consumers is beyond the scope of the current paper and, therefore, could also be a promising future research direction (Laffont, 1994). 


\section{References}

Anderson, E.J., A.B. Philpott, H. Xu (2007). Modelling the effects of interconnection between electricity markets. Math. Methods Oper. Res. 65(1):1-26.

Baringo, L., A.J. Conejo (2013). Transmission and wind power investment. IEEE Trans. Power Syst. 27(2):885-893.

Barnett, A.H. (1980). The Pigouvian tax rule under monopoly. Amer. Econ. Rev. 70(5):1037-1041.

Boomsma, T.K., N. Meade, S.-E. Fleten (2012). Renewable energy investments under different support schemes: A real options approach. Eur. J. Oper. Res. 220(1):225-237.

Borenstein, S., J.B. Bushnell, S. Stoft (2000). The competitive effects of transmission capacity in a deregulated electricity industry. RAND J. Econ. 31(2):294-325.

Borgen, H. (2016). Statnett og vindkraft: Utfordrende koordinering innenfor knappe tidsrammer (Statnett and wind power: Challenging coordination within a short time frame). NVEs vindkraftseminar (The Norwegian Water Resources and Energy Directorate's seminar on wind power), 1314 June 2016, http://webfileservice.nve.no/API/PublishedFiles/Download/201602121/ 1784331.

Bushnell, J.B., S.P. Holland, J.E. Hughes, C.R. Knittel (2017). Strategic policy choice in state-level regulation: the EPA's Clean Power Plan. Amer. Econ. J.: Econ. Pol. 9(2):57-90.

California ISO (2018). Annual Report on Market Issues and Performance - 2017. http://www . caiso.com/Documents/2017AnnualReportonMarketIssuesandPerformance.pdf, Folsom, CA.

Chao, H.-P., S. Peck (1996). A market mechanism for electric power transmission. J. Reg. Econ. $10(1): 25-59$.

Downward, A. (2010). Carbon charges in electricity markets with strategic behavior and transmission. The Ener. J. 31(4):159-166.

European Commission (2009). Directive 2009/28/EC of the European Parliament and of the Council. http://eur-lex.europa.eu/legal-content/EN/TXT/?uri=CELEX:32009L0028, Brussels, Belgium.

European Commission (2018). EU Emissions Trading System: Free Allocation of Allowances. https://ec.europa.eu/clima/policies/ets/allowances_en, Brussels, Belgium.

European Network of Transmission System Operators for Electricity (2018). https: //www . entsoe . eu/about-entso-e/inside-entso-e/member-companies/Pages/default.aspx, Brussels, Belgium. 
Federal Energy Regulatory Commission (2011). Transmission Planning and Cost Allocation by Transmission Owning and Operating Public Utilities. Docket No. RM10-23-000; Order No. 1000. https://www.ferc.gov/whats-new/comm-meet/2011/072111/E-6.pdf, Washington, DC.

Fitzpatrick, D., D. Barrett (2013). J.P. Morgan faces new probe on energy trades. The Wall Street Journal, August 19, 2013, https://www.wsj.com/articles/ jp-morgan-faces-new-probe-on-energy-trades-1376947109?tesla=y.

Fleten, S.-E., A.M. Heggedal, A.S. Siddiqui (2011). Transmission capacity between Norway and Germany: a real options analysis. J. Ener. Markets 4(1):121-147.

Gabriel, S.A., A.J. Conejo, J.D. Fuller, B.F. Hobbs, C. Ruiz (2012). Complementarity Modeling in Energy Markets. Springer, Germany.

Garver, L.L. (1970). Transmission network estimation using linear programming. IEEE Trans. Power App. 83 Syst. PAS-89(7):1688-1697.

Hobbs, B.F. (1995). Optimization methods for electric utility resource planning. Eur. J. Oper. Res. 83(1):1-20.

Hobbs, B.F. (2001). Linear complementarity models of Nash-Cournot competition in bilateral and POOLCO power markets. IEEE Trans. Power Syst. 16(2):194-202.

Hobbs, B.F. (2012). Transmission planning and pricing for renewables: lessons from elsewhere. 2012 IEEE Power and Energy Society General Meeting, 22-26 July 2012, San Diego, CA.

Hogan, W.W. (1992). Contract networks for electric power transmission. J. Reg. Econ. 4(3):211-242.

Huppmann, D., S.A. Siddiqui (2018). An exact solution method for binary equilibrium problems with compensation and the power market uplift problem. Eur. J. Oper. Res. 266(2):622-638.

Joskow, P.L. (2008). Lessons learned from electricity market liberalization. The Ener. J. 29(1):9-42.

Joskow, P.L., J. Tirole (2005). Merchant transmission investment. J. Ind. Econ. 53(2):233-264.

Just, S., C. Weber (2015). Strategic behavior in the German balancing energy mechanism: incentives, evidence, costs and solutions. J. Reg. Econ. 48(2):218-243.

Laffont, J.J. (1994). Regulation of pollution with asymmetric information. Nonpoint Source Pollution Regulation: Issues and Analysis, C. Dosi and T. Tomasi, eds., Kluwer Academic Publishers, Dordrecht.

Kunz, F. (2013). Improving congestion management: how to facilitate the integration of renewable generation in Germany. The Ener. J. 34:55-78. 
Martin, R. (2016). In Texas oil country, wind is straining the grid. MIT Technology Review, August 6, 2016. https://www.technologyreview.com/s/602112/ in-texas-oil-country-wind-is-straining-the-grid/, Cambridge, MA.

Maurovich-Horvat, L., T.K. Boomsma, A.S. Siddiqui (2015). Transmission and wind investment in a deregulated electricity industry. IEEE Trans. Power Syst. 30(3):1633-1643.

Murphy, F., Y. Smeers (2010). On the impact of forward markets on investments in oligopolistic markets with reference to electricity. Oper. Res. 58(3):515-528.

Nash, S.G. and A. Sofer (1995). Linear and Nonlinear Programming. McGraw-Hill College, New York.

Nasiri, F., G. Zaccour (2010). Renewable portfolio standard policy: a game-theoretic analysis. INFOR 48(4):251-260.

Organization for Cross-regional Coordination of Transmission Operators, Japan (2017). Annual Report. Fiscal Year 2016. https://www.occto.or.jp/en/information_disclosure/annual_ report/files/annual_report_FY2016.pdf, Tokyo, Japan.

PJM Interconnection (2018). PJM State of the Market - 2017. http://www .monitoringanalytics . com/reports/PJM_State_of_the_Market/2017.shtml, Eagleville, PA.

Requate, T. (2006). Environmental policy under imperfect competition. The International Yearbook of Environmental and Resource Economics 2006/200\%: A Survey of Current Issues, T. Tietenberg and H. Folmer, eds., pp. 120-187.

Rintamäki, T., A.S. Siddiqui, A. Salo (2016). How much is enough? Optimal support payments in a renewable-rich power system. Ener. 117:300-313.

Rintamäki, T., A.S. Siddiqui, A. Salo (2017). Does renewable energy generation decrease the volatility of electricity prices? An analysis of Denmark and Germany. Ener. Econ. 62:270-282.

Sauma, E.E., S.S. Oren (2006). Proactive planning and valuation of transmission investments in restructured electricity markets. J. Reg. Econ. 30:261-290.

Sauma, E.E., S.S. Oren (2009). Do generation firms in restructured electricity markets have incentives to support social-welfare-improving transmission investments? Ener. Econ. 31:676-689.

Schweppe, F.C., M.C. Caramanis, R.D. Tabors, R.E. Bohn (1988). Spot Pricing of Electricity. Springer, U.S.

Siddiqui, A.S., M. Tanaka, Y. Chen (2016). Are targets for renewable portfolio standards too low? The impact of market structure on energy policy. Eur. J. Oper. Res. 250(1):328-341.

Tanaka, M., Y. Chen (2013). Market power in renewable portfolio standards. Ener. Econ. 39:187196. 
Tangerås, T., J. Mauritzen (2018). Real-time versus day-ahead market power in a hydro-based electricity market. J. Ind. Econ., forthcoming.

Vara, V. (2016). The energy interstate. The Atlantic, June 2016 issue, http://www. theatlantic. com/magazine/archive/2016/06/the-energy-interstate/480756/.

von Hirschhausen, C. (2014). The German 'Energiewende'-an introduction. Econ. Ener. Env. Pol. $3: 1-12$.

Wilson, R.B. (2002). Architecture of power markets. Econometrica 70(4): 1299-1340.

\section{Appendix: Proofs of Propositions}

We denote $I=C_{R}-C_{F}-C_{T}$ and $G=C_{R}-C_{F}+C_{T}$, where $G>0$ as $C_{R}>C_{F}$.

\section{Proof of Proposition 1}

Case 1: At the optimum, $-\left[A_{R}-B_{R}\left(y_{R}+t\right)\right]+C_{R}=\frac{B_{R} B_{F} I-D\left[B_{F}\left(A_{R}-C_{R}\right)+B_{R}\left(A_{F}-C_{R}+C_{T}\right)\right]}{\left(B_{F}+B_{R}\right) D+B_{R} B_{F}}$ with regard to KKT condition (6). This needs to be positive for Case 1 with $\bar{y}_{R}=0$. The denominator and the expression in the square bracket in the numerator are positive, noting that $A_{F}>A_{R}>C_{R}>C_{F}$. Thus, $I$ needs to be positive for Case 1 to occur, i.e., $C_{R}>$ $C_{F}+C_{T}$. We next calculate the derivative of each variable with respect to $D$. We obtain $\frac{\partial \bar{k}}{\partial D}=-\frac{B_{F}\left[B_{R}\left(A_{F}-C_{F}\right)+B_{F}\left(A_{R}-C_{R}+I\right)\right]}{\left[\left(B_{F}+B_{R}\right) D+B_{R} B_{F}\right]^{2}}<0$ and $\frac{\partial \bar{y}_{F}}{\partial D}=-\frac{\left(B_{F}+B_{R}\right)\left[B_{R}\left(A_{F}-C_{F}\right)+B_{F}\left(A_{R}-C_{R}+I\right)\right]}{\left[\left(B_{F}+B_{R}\right) D+B_{R} B_{F}\right]^{2}}<0$.

Case 2: At the optimum, $\bar{y}_{F}=\frac{I}{D}$, which needs to be positive for Case 2. Thus, $I$ needs to be positive for Case 2 to occur. We obtain $\frac{\partial \bar{k}}{\partial D}=-\frac{I}{D^{2}}<0, \frac{\partial \bar{y}_{F}}{\partial D}=-\frac{I}{D^{2}}<0$, and $\frac{\partial \bar{y}_{R}}{\partial D}=\frac{I}{D^{2}}>0$.

Case 3: Simple calculation yields $\frac{\partial \bar{y}_{F}}{\partial D}=-\frac{A_{F}-C_{F}}{\left(B_{F}+D\right)^{2}}<0$ and $\frac{\partial \bar{y}_{R}}{\partial D}=0$.

Case 4: We have $\frac{\partial \bar{k}}{\partial D}=\frac{G}{D^{2}}>0, \frac{\partial \bar{y}_{F}}{\partial D}=-\frac{G}{D^{2}}<0$, and $\frac{\partial \bar{y}_{R}}{\partial D}=\frac{G}{D^{2}}>0$.

Proof of Proposition 2 Differentiating the damage cost $\frac{1}{2} D \bar{y}_{F}^{2}$ with respect to $D$ at the optimum yields $-\frac{I^{2}}{2 D^{2}}<0$ and $-\frac{G^{2}}{2 D^{2}}<0$ in Cases 2 and 4 , respectively.

\section{Proof of Proposition 3}

Case 1: At the optimum, $-\left[A_{R}-B_{R}\left(y_{R}+t\right)\right]+C_{R}=\frac{B_{R} B_{F} I-D\left[B_{F}\left(A_{R}-C_{R}\right)+B_{R}\left(A_{F}-C_{F}\right)\right]}{B_{F}\left(D+B_{R}\right)}$ with regard to KKT condition (17). This needs to be positive for Case 1 with $\hat{y}_{R}=0$. The denominator and the expression in the square bracket in the numerator are positive. Thus, $I$ needs to be positive for Case 1 to occur. We next calculate the derivative of each variable with respect to $D$. We obtain $\frac{\partial \hat{k}}{\partial D}=-\frac{B_{R}\left(A_{F}-C_{F}\right)+B_{F}\left(A_{R}-C_{R}+I\right)}{B_{F}\left(D+B_{R}\right)^{2}}<0$ and $\frac{\partial \hat{y}_{F}}{\partial D}=\hat{y}_{F}^{\prime}(k) \frac{\partial \hat{k}}{\partial D}=\frac{\partial \hat{k}}{\partial D}<0$.

Case 2: At the optimum, $\hat{y}_{F}=\frac{I}{D}$, which needs to be positive for Case 2. Thus, $I$ needs to be positive for Case 2 to occur. We obtain $\frac{\partial \hat{k}}{\partial D}=-\frac{I}{D^{2}}<0, \frac{\partial \hat{y}_{F}}{\partial D}=\hat{y}_{F}^{\prime}(k) \frac{\partial \hat{k}}{\partial D}=\frac{\partial \hat{k}}{\partial D}<0$, and $\frac{\partial \hat{y}_{R}}{\partial D}=\hat{y}_{R}^{\prime}(k) \frac{\partial \hat{k}}{\partial D}=-\frac{\partial \hat{k}}{\partial D}>0$.

Case 3: Simple calculation yields $\frac{\partial \hat{y}_{F}}{\partial D}=\frac{\partial \hat{y}_{R}}{\partial D}=0$. 
Proof of Proposition 4 Differentiating the damage cost $\frac{1}{2} D \hat{y}_{F}^{2}$ with respect to $D$ at the optimum yields $-\frac{I^{2}}{2 D^{2}}<0$ and $\frac{\left(A_{F}-C_{F}\right)^{2}}{2 B_{F}^{2}}>0$ in Cases 2 and 3 , respectively.

\section{Proof of Proposition 5}

Case 1: From KKT condition $(24),-\left[A_{R}-B_{R}\left(2 y_{R}+t\right)\right]+C_{R}=\frac{H-D\left[B_{F}\left(A_{R}-C_{R}\right)+B_{R}\left(A_{F}-C_{F}\right)\right]}{B_{F}\left(B_{F}+4 B_{R}+D\right)}$ at the optimum, which needs to be positive for Case 1 with $y_{R}^{*}=0$. The denominator and the expression in the square bracket in the numerator are positive. Let

$$
H=B_{F}\left[B_{F}\left(C_{R}-A_{R}\right)+B_{R}\left(C_{R}-A_{F}-C_{T}\right)+3 B_{R} I\right]
$$

where the expressions in the first two parentheses are negative. Thus, $I$ needs to be positive for Case 1 to occur. We next calculate the derivative of each variable with respect to $D$. We obtain $\frac{\partial k^{*}}{\partial D}=-\frac{4\left[B_{R}\left(A_{F}-C_{F}\right)+B_{F}\left(A_{R}-C_{R}+I\right)\right]}{B_{F}\left(B_{F}+4 B_{R}+D\right)^{2}}<0$ and $\frac{\partial y_{F}^{*}}{\partial D}=y_{F}^{* \prime}(k) \frac{\partial k^{*}}{\partial D}=\frac{1}{2} \frac{\partial k^{*}}{\partial D}<0$.

Case 2: From Table $1, k^{*}=\frac{B_{F}\left(A_{R}-A_{F}-C_{T}+3 F\right)-D\left(A_{F}-C_{F}\right)}{B_{F}\left(B_{F}+B_{R}+D\right)}$ at the optimum, which needs to be positive for Case 2. The denominator is positive. In the numerator, $A_{R}-A_{F}-C_{T}<0$ and $A_{F}-C_{F}>0$. Thus, $I$ needs to be positive for Case 2 to occur. We obtain $\frac{\partial k^{*}}{\partial D}=$ $-\frac{B_{F}\left(A_{R}-C_{R}+4 F\right)+B_{R}\left(A_{F}-C_{F}\right)}{B_{F}\left(B_{F}+B_{R}+D\right)^{2}}<0, \frac{\partial y_{F}^{*}}{\partial D}=y_{F}^{* \prime}(k) \frac{\partial k^{*}}{\partial D}=\frac{1}{2} \frac{\partial k^{*}}{\partial D}<0$, and $\frac{\partial y_{R}^{*}}{\partial D}=y_{R}^{* \prime}(k) \frac{\partial k^{*}}{\partial D}=-\frac{1}{2} \frac{\partial k^{*}}{\partial D}>$ 0.

Case 3: Simple calculation yields $\frac{\partial y_{F}^{*}}{\partial D}=\frac{\partial y_{R}^{*}}{\partial D}=0$.

Case 4: We have $\frac{\partial k^{*}}{\partial D}=\frac{B_{F}\left(A_{R}-C_{R}+4 G\right)+B_{R}\left(A_{F}-C_{F}\right)}{B_{F}\left(B_{F}+B_{R}+D\right)^{2}}>0, \frac{\partial y_{F}^{*}}{\partial D}=y_{F}^{* \prime}(k) \frac{\partial k^{*}}{\partial D}=-\frac{1}{2} \frac{\partial k^{*}}{\partial D}<0$, and $\frac{\partial y_{R}^{*}}{\partial D}=y_{R}^{* \prime}(k) \frac{\partial k^{*}}{\partial D}=\frac{1}{2} \frac{\partial k^{*}}{\partial D}>0$.

Proof of Proposition 6 Differentiating the damage cost $\frac{1}{2} D y_{F}^{* 2}$ with respect to $D$ at the optimum yields $\frac{\left(A_{F}-C_{F}\right)^{2}}{8 B_{F}^{2}}>0$ in Case 3 .

Proof of Proposition 7 By using the envelope theorem, we calculate the partial derivative of the Lagrangian for the QP problem (1)-(4) with respect to $D$ evaluated at the optimum. This gives $-\frac{1}{2} \bar{y}_{F}^{2}<0$ at the optimum for every case under CP. Thus, the value function (i.e., maximised social welfare here) is decreasing in $D$ under CP. In a similar way using the envelope theorem, we can easily verify $-\frac{1}{2} \hat{y}_{F}^{2}<0$ and $-\frac{1}{2} y_{F}^{* 2}<0$ at the optimum for every case under $\mathrm{PC}$ and CO, respectively.

Proof of Proposition 8 The CP welfare-maximisation problem, (1)-(4), is equivalent to the following decomposed problem:

$$
\begin{array}{cl}
\max _{\{k \geq 0\} \cup \Omega \cup \Xi} & \text { Equation (1) } \\
\text { s.t. } & \max _{\Omega} \text { Equation (1) } \\
& \text { s.t. Equations (2)-(4) }
\end{array}
$$


Since $\max _{\Omega}\{$ Equation (1) | Equations (2)-(4) $\}$ is a convex programming problem, it can be replaced by its KKT conditions as follows:

$$
\begin{array}{cl}
\max _{\{k \geq 0\} \cup \Omega \cup \Xi} & \text { Equation (1) } \\
\text { s.t. } & \text { Equations (5)-(6), (8)-(12) }
\end{array}
$$

With our assumption of $\beta_{R}=0$ and $\beta_{F}=0$, this is equivalent to the TSO's problem (31) under PC with a full carbon charge, $E=1$.

\section{Proof of Proposition 9}

Case 1: We calculate $-\left[A_{R}-B_{R}\left(y_{R}+t\right)\right]+C_{R}$ at the optimum regarding the KKT condition with respect to $y_{R}$. This needs to be positive for Case 1 with $\hat{y}_{R}=0$. The denominator is positive, while the numerator is decomposed into negative terms and $B_{R} B_{F} I\left(B_{F}+2 E D\right)$. Thus, $I$ needs to be positive for Case 1 to occur. We then calculate $\frac{\partial \hat{k}}{\partial E}$. The denominator is a squared term, while the numerator is $2 B_{F} D^{2}(1-E)\left(B_{F}+E D\right)\left[B_{R}\left(A_{F}-C_{F}\right)+B_{F}\left(A_{R}-C_{R}+I\right)\right]>0$. Hence, $\frac{\partial \hat{k}}{\partial E}>0$.

Case 2: At the optimum, $\hat{y}_{F}=\frac{\left(B_{F}+E D\right) I}{D\left(B_{F}+E^{2} D\right)}$, which needs to be positive for Case 2. Thus, $I$ needs to be positive for Case 2 to occur. We then obtain $\frac{\partial \hat{k}}{\partial E}=\frac{2(1-E)\left(B_{F}+E D\right) I}{\left(B_{F}+E^{2} D\right)^{2}}>0$.

Case 4: We have $\frac{\partial \hat{k}}{\partial E}=-\frac{2(1-E)\left(B_{F}+E D\right) G}{\left(B_{F}+E^{2} D\right)^{2}}<0$.

\section{Proof of Proposition 10}

Case 1: We calculate $-\left[A_{R}-B_{R}\left(2 y_{R}+t\right)\right]+C_{R}$ at the optimum regarding KKT condition with respect to $y_{R}$. This needs to be positive for Case 1 with $y_{R}^{*}=0$. The denominator is positive, while the numerator is decomposed into negative terms and $B_{R} B_{F} I\left(3 B_{F}+2 E D\right)$. Thus, $I$ needs to be positive for Case 1 to occur. We then calculate $\frac{\partial k^{*}}{\partial E}$. The denominator is a squared term, and the numerator is $2 B_{F} D\left[(1-E) D-B_{F}\right]\left(2 B_{F}+E D\right)\left[B_{R}\left(A_{F}-C_{F}\right)+B_{F}\left(A_{R}-C_{R}+I\right)\right]$. Hence, $\frac{\partial k^{*}}{\partial E}>0$ if and only if $(1-E) D-B_{F}>0$, or $E>1-\frac{B_{F}}{D}$.

Case 2: We calculate $k^{*}$ at the optimum, which needs to be positive for Case 2. The denominator is positive, while the numerator is decomposed into negative terms and $4 B_{F} I\left(3 B_{F}+\right.$ $2 E D)$. Thus, $I$ needs to be positive for Case 2 to occur. We then calculate $\frac{\partial k^{*}}{\partial E}$. The denominator is a squared term, while the numerator is $8 B_{F} D\left[(1-E) D-B_{F}\right]\left(2 B_{F}+E D\right)$ $\left[B_{R}\left(A_{F}-C_{F}\right)+B_{F}\left(A_{R}-C_{R}\right)+4 B_{F} I\right]$. Hence, $\frac{\partial k^{*}}{\partial E}>0$ if and only if $E>1-\frac{B_{F}}{D}$.

Case 4: We calculate $\frac{\partial k^{*}}{\partial E}$. The denominator is a squared term, while the numerator is $-8 B_{F} D$ $\left[(1-E) D-B_{F}\right]\left(2 B_{F}+E D\right)\left[B_{R}\left(A_{F}-C_{F}\right)+B_{F}\left(A_{R}-C_{R}\right)+4 B_{F} G\right]$. Hence, $\frac{\partial k^{*}}{\partial E}<0$ if and only if $E>1-\frac{B_{F}}{D}$. 
Proof of Proposition 11 From KKT condition (30), $A_{F}-B_{F}\left(y_{F}-t\right)-C_{F}-E D y_{F}=0$ since $\hat{y}_{F}>0$ in Cases 1-4. We thus derive $\hat{y}_{F}(t, E)=\frac{A_{F}-C_{F}+B_{F} t}{B_{F}+E D}$. For all Cases 1-4, the TSO's problem can be generally expressed as follows using variable $t$ :

$$
\begin{aligned}
\max _{t} & A_{F}\left(\hat{y}_{F}(t, E)-t\right)-\frac{1}{2} B_{F}\left(\hat{y}_{F}(t, E)-t\right)^{2}-C_{F} \hat{y}_{F}(t, E) \\
& +A_{R}\left(\hat{y}_{R}(t)+t\right)-\frac{1}{2} B_{R}\left(\hat{y}_{R}(t)+t\right)^{2}-C_{R} \hat{y}_{R}(t)-C_{T}|t|-\frac{1}{2} D \hat{y}_{F}(t, E)^{2}
\end{aligned}
$$

where $t=k$ and $\hat{y}_{R}(t)=0$ in Case $1, t=k$ in Case 2, $t=k=0$ in Case 3, and $t=-k$ in Case 4. We calculate the partial derivative of the objective function with respect to $E$ evaluated at the optimum, i.e., for $k=\hat{k}$ and $t=\hat{t}$. This gives $\frac{\partial \hat{y}_{F}}{\partial E}\left[A_{F}-B_{F}\left(\hat{y}_{F}-\hat{t}\right)-C_{F}-D \hat{y}_{F}\right]$. From $A_{F}-B_{F}\left(\hat{y}_{F}-\hat{t}\right)-C_{F}-E D \hat{y}_{F}=0$, we have $A_{F}-B_{F}\left(\hat{y}_{F}-\hat{t}\right)-C_{F}-D \hat{y}_{F}=-(1-E) D \hat{y}_{F}<0$. For Cases 1-3, $\frac{\partial \hat{y}_{F}}{\partial E}=-\frac{D\left(A_{F}-C_{F}+B_{F} \hat{t}\right)}{\left(B_{F}+E D\right)^{2}}=-\frac{D\left(A_{F}-C_{F}+B_{F} \hat{k}\right)}{\left(B_{F}+E D\right)^{2}}<0$. For Case 4, $\frac{\partial \hat{y}_{F}}{\partial E}=-\frac{D\left(A_{F}-C_{F}+B_{F} \hat{t}\right)}{\left(B_{F}+E D\right)^{2}}=$ $-\frac{D\left(A_{F}-C_{F}-B_{F} \hat{k}\right)}{\left(B_{F}+E D\right)^{2}}<0$ since $A_{F}-C_{F}-B_{F} \hat{k}=\left(B_{F}+E D\right) \hat{y}_{F}>0$. Therefore, $\frac{\partial s \hat{w}}{\partial E}>0$ by the envelope theorem.

Proof of Proposition 12 From KKT condition (32), $A_{F}-B_{F}\left(2 y_{F}-t\right)-C_{F}-E D y_{F}=0$ since $y_{F}^{*}>0$ in Cases $1-4$. We, thus, derive $y_{F}^{*}(t, E)=\frac{A_{F}-C_{F}+B_{F} t}{2 B_{F}+E D}$. For all Cases $1-4$, the TSO's problem can be generally expressed as follows using variable $t$ :

$$
\begin{aligned}
\max _{t} & A_{F}\left(y_{F}^{*}(t, E)-t\right)-\frac{1}{2} B_{F}\left(y_{F}^{*}(t, E)-t\right)^{2}-C_{F} y_{F}^{*}(t, E) \\
& +A_{R}\left(y_{R}^{*}(t)+t\right)-\frac{1}{2} B_{R}\left(y_{R}^{*}(t)+t\right)^{2}-C_{R} y_{R}^{*}(t)-C_{T}|t|-\frac{1}{2} D y_{F}^{*}(t, E)^{2}
\end{aligned}
$$

where $t=k$ and $y_{R}^{*}(t)=0$ in Case $1, t=k$ in Case $2, t=k=0$ in Case 3 , and $t=-k$ in Case 4. We calculate the partial derivative of the objective function with respect to $E$ evaluated at the optimum, i.e., for $k=k^{*}$ and $t=t^{*}$. This gives $\frac{\partial y_{F}^{*}}{\partial E}\left[A_{F}-B_{F}\left(y_{F}^{*}-t^{*}\right)-C_{F}-D y_{F}^{*}\right]$. From $A_{F}-$ $B_{F}\left(2 y_{F}^{*}-t^{*}\right)-C_{F}-E D y_{F}^{*}=0$, we have $A_{F}-B_{F}\left(y_{F}^{*}-t\right)-C_{F}-D y_{F}^{*}=\left[B_{F}-(1-E) D\right] y_{F}^{*}$. This is positive if and only if $E>1-\frac{B_{F}}{D}$. For Cases 1-3, $\frac{\partial y_{F}^{*}}{\partial E}=-\frac{D\left(A_{F}-C_{F}+B_{F} t^{*}\right)}{\left(2 B_{F}+E D\right)^{2}}=-\frac{D\left(A_{F}-C_{F}+B_{F} k^{*}\right)}{\left(2 B_{F}+E D\right)^{2}}<$ 0 . For Case $4, \frac{\partial y_{F}^{*}}{\partial E}=-\frac{D\left(A_{F}-C_{F}+B_{F} t^{*}\right)}{\left(2 B_{F}+E D\right)^{2}}=-\frac{D\left(A_{F}-C_{F}-B_{F} k^{*}\right)}{\left(2 B_{F}+E D\right)^{2}}<0$ since $A_{F}-C_{F}-B_{F} k^{*}=\left(2 B_{F}+\right.$ $E D) y_{F}^{*}>0$. Therefore, if $E>1-\frac{B_{F}}{D}$, then $\frac{\partial s w^{*}}{\partial E}<0$, and vice versa, by the envelope theorem.

Proof of Proposition 13 As shown in Proposition 11, sîw $(E)$ monotonically increases in $E$ under PC. Hence, $s \hat{w}(E)$ is maximised at $E=1$ under PC.

Proof of Proposition 14 As shown in Proposition 12, sw $w^{*}(E)$ increases in $E$ in the range of $E<1-\frac{B_{F}}{D}$, while it decreases in $E$ in the range of $E>1-\frac{B_{F}}{D}$. If $B_{F}<D$, then $0<1-\frac{B_{F}}{D}<1$, and, thus, $s w^{*}(E)$ is maximised at $E=1-\frac{B_{F}}{D}<1$. If $B_{F} \geq D$, then $1-\frac{B_{F}}{D} \leq 0$, and, thus, $s w^{*}(E)$ is maximised at $E=0$. 